\title{
Unveiling the environment and faint features of the isolated galaxy CIG 96 with deep optical and $\mathrm{HI}$ observations ${ }^{\star}$
}

\author{
P. Ramírez-Moreta ${ }^{1}$, L. Verdes-Montenegro $^{1}$, J. Blasco-Herrera ${ }^{1}$, S. Leon ${ }^{2}$, A. Venhola ${ }^{3,4}$, M. Yun ${ }^{5}$, V. Peris ${ }^{6}$, \\ R. Peletier ${ }^{3}$, G. Verdoes Kleijn ${ }^{3}$, E. Unda-Sanzana ${ }^{11}$, D. Espada ${ }^{7,8}$, A. Bosma ${ }^{9}$, E. Athanassoula ${ }^{9}$, \\ M. Argudo-Fernández ${ }^{11}$, J. Sabater ${ }^{10}$, J. C. Muñoz-Mateos ${ }^{2}$, M. G. Jones ${ }^{1}$, W. Huchtmeier ${ }^{12}$, J. E. Ruiz ${ }^{1}$, \\ J. Iglesias-Páramo ${ }^{1,13}$, M. Fernández-Lorenzo ${ }^{1}$, J. Beckman ${ }^{14}$, S. Sánchez-Expósito ${ }^{1}$, and J. Garrido ${ }^{1}$ \\ ${ }^{1}$ Instituto de Astrofísica de Andalucía (IAA-CSIC), Glorieta de la Astronomía s/n, 18008 Granada, Spain \\ e-mail: prm@iaa.es, pramirezmoreta@gmail.com \\ 2 Joint ALMA Observatory - ESO, Av. Alonso de Córdova, 3104 Santiago, Chile \\ 3 Kapteyn Instituut, Postbus 800, 9700 AV Groningen, The Netherlands \\ 4 Astronomy Research Unit, University of Oulu, 90014 Oulu, Finland \\ 5 Department of Astronomy, University of Massachusetts-Amherst, LGRT-B 522710 North Pleasant Street, Amherst, MA, USA \\ 6 Observatori Astronòmic de la Universitat de València, Catedrático José Beltrán, 2, 46980 Paterna, Spain \\ 7 National Astronomical Observatory of Japan (NAOJ), 2-21-1 Osawa, Mitaka 181-8588, Tokyo, Japan \\ 8 The Graduate University for Advanced Studies (SOKENDAI), 2-21-1 Osawa, Mitaka 181-0015, Tokyo, Japan \\ 9 Aix-Marseille Université, CNRS, CNES, LAM, Marseille, France \\ ${ }^{10}$ Institute for Astronomy, University of Edinburgh, Royal Observatory, Blackford Hill, Edinburgh EH9 3HJ, UK \\ 11 Universidad de Antofagasta, Unidad de Astronomía, Facultad Cs. Básicas, Av. U. de Antofagasta 02800, Antofagasta, Chile \\ 12 Max-Planck-Institut für Radioastronomie, Auf dem Hügel 69, 53121 Bonn, Germany \\ 13 Estación Experimental de Zonas Áridas (CSIC), Ctra. de Sacramento s/n, La Cañada de San Urbano, 04120 Almería, Spain \\ 14 Instituto de Astrofísica de Canarias, c/Vía Láctea, s/n, 38205 La Laguna, Tenerife, Spain
}

Received 1 May 2018 / Accepted 30 July 2018

\section{ABSTRACT}

\begin{abstract}
Context. Asymmetries in atomic hydrogen (HI) in galaxies are often caused by the interaction with close companions, making isolated galaxies an ideal framework to study secular evolution. The AMIGA project has demonstrated that isolated galaxies show the lowest level of asymmetry in their HI integrated profiles compared to even field galaxies, yet some present significant asymmetries. CIG 96 (NGC 864) is a representative case reaching a $16 \%$ level.

Aims. Our aim is to investigate the HI asymmetries of the spiral galaxy CIG 96 and what processes have triggered the star-forming regions observed in the XUV pseudo-ring.

Methods. We performed deep optical observations at CAHA1.23m, CAHA2.2m and VST (OmegaCAM wide-field camera) telescopes. We reach surface brightness (SB) limits of $\mu_{\mathrm{CAHA} 2.2 \mathrm{~m}}=27.5 \mathrm{mag} \operatorname{arcsec}^{-2}$ (Cousins $R$ ) and $\mu_{\mathrm{VST}}=28.7 \mathrm{mag} \mathrm{arcsec}^{-2}$ (SDSS $r$ ) that show the XUV pseudo-ring of the galaxy in detail. Additionally, a wavelet filtering of the HI data cube from our deep observations with VLA/EVLA telescope allowed us to reach a column density of $N_{\mathrm{HI}}=8.9 \times 10^{18} \mathrm{~cm}^{-2}(5 \sigma)\left(28^{\prime \prime} \times 28^{\prime \prime}\right.$ beam $)$, lower than in any isolated galaxy.

Results. We confirm that the HI of CIG 96 extends farther than $4 \times r_{25}$ in all directions. Furthermore, we detect for the first time two gaseous structures $\left(\sim 10^{6} M_{\odot}\right)$ in the outskirts. The SDSS $g-r$ colour index image from CAHA1.23m shows extremely blue colours in certain regions of the pseudo-ring where $N_{\mathrm{HI}}>8.5 \times 10^{20} \mathrm{~cm}^{-2}$, whereas the rest show red colours. Galactic cirrus contaminate the field, setting an unavoidable detection limit at $28.5 \mathrm{mag} \mathrm{arcsec}^{-2}$ (SDSS $r$ ).

Conclusions. At the current SB and $N_{\mathrm{HI}}$ levels, we detect no stellar link within $1^{\circ} \times 1^{\circ}$ or gaseous link within $40^{\prime} \times 40^{\prime}$ between CIG 96 and any companion. The isolation criteria rule out interactions with other similar-sized galaxies for at least $\sim 2.7 \mathrm{Gyr}$. Using existing stellar evolution models, the age of the pseudo-ring is estimated at $1 \mathrm{Gyr}$ or older. Undetected previously accreted companions and cold gas accretion remain as the main hypothesis to explain the optical pseudo-ring and HI features of CIG 96.
\end{abstract}

Key words. galaxies: individual: NGC 864 - galaxies: spiral - galaxies: structure - galaxies: evolution galaxies: kinematics and dynamics - radio lines: galaxies

\section{Introduction}

Most galaxies in the nearby universe are either interacting with or gravitationally bound to nearby companions. Such events are

\footnotetext{
$\star$ The reduced images and datacubes are only available at the CDS via anonymous ftp to cdsarc.u-strasbg.fr (130.79.128.5) or via http://cdsarc.u-strasbg.fr/viz-bin/qcat?]/A+A/619/ A163
}

directly responsible for a continuous change in their structural, dynamical and chemical properties (Toomre 1977). A wide set of observed and, broadly, understood effects of such interactions (e.g. quenching or enhancement of the stellar formation, gaseous plumes and bridges, tidal streams, etc.) constitute some of the main drivers of the evolution of galaxies. Such interactions may prevail over the internal processes, hiding or even disrupting the key inner evolutionary mechanisms of each particular 
galaxy that, in the absence of large companions, might otherwise dominate its evolution. The bars present in nearly two-thirds of the spiral galaxies (e.g. Buta et al. 2015), whether initially from external or internal origin, are among these inner elements that can crucially affect the evolution of the galaxy from their bulges or pseudo-bulges out to the outer Lindblad resonance in their external regions (e.g. Kormendy \& Kennicutt 2004; Buta et al. 2005; Fernández Lorenzo et al. 2014). Additionally, the results from other cosmologically motivated studies point out that the interaction of the galaxies with dark matter halos might result in perturbations of the disc (e.g. Kazantzidis et al. 2008, 2009). Isolated galaxies, if selected with strict and robust criteria, constitute an ideal framework to study the secular evolution of galaxies since we can exclude the possibility of interactions with large companions. The Analysis of the interstellar Medium of Isolated GAlaxies (AMIGA) project ${ }^{1}$ (Verdes-Montenegro et al. 2005) was designed to perform a multi-wavelength study of a large sample of galaxies selected with strict isolation criteria from the Catalog of Isolated Galaxies $\left(\mathrm{CIG}^{2}, 1051\right.$ galaxies, Karachentseva 1973).

With respect to the isolation level, a plethora of references to different definitions and selection criteria may be found throughout the literature of the last 40 years, (e.g. all references in Verdes-Montenegro et al. 2005 or Muldrew et al. 2012 among others). As part of the AMIGA project, this work makes use of its isolation criteria and parameters (local number density $\eta_{k}$ and tidal force estimation $Q$ ) in the version by Verley et al. (2007) revised later by Argudo-Fernández et al. (2013, 2014). Both isolation parameters are defined in depth in the discussion of the environment (see Sect. 5.1).

The results of the project are that variables expected to be enhanced by interactions are lower in isolated galaxies than in any other sample (e.g. $L_{\mathrm{FIR}}$, Lisenfeld et al. 2007, radio continuum emission, optical symmetry, Verdes-Montenegro et al. 2010 and references therein, active galactic nucleus (AGN) rate, Sabater et al. 2012). Among these, one specific result is especially significant in the context of the present work: the asymmetry level of the atomic gas (HI) integrated profiles of the CIG galaxies is also lower than any other sample, including field galaxies (Espada et al. 2011b, see Jones et al. 2018 for a full characterisation of the HI content of AMIGA sample). However, a number of galaxies show unusually high levels of asymmetry (up to $50 \%$ ), the causes of which remain unknown.

If asymmetries can only be generated by interactions, lopsidedness in an isolated galaxy such as CIG 96 (NGC 864) should not be observed. However, previous data from Green Bank as well as VLA observatories show a large HI envelope beyond $2 \times r_{25}$ (q.v. Table 1) that has an asymmetry level of $16 \%$ in its HI integrated profile (Espada et al. 2005).

Espada et al. (2011a) report on a partial XUV ring (hereafter the pseudo-ring, see Sect. 4.1) seen in near-UV (NUV) and farUV (FUV) GALEX data, and located at $1.5-2 \times r_{25}$. This pseudoring shows patchy regions with star formation (SF). It is not clear that such features can develop in galaxies free from interactions. In this paper we present additional data on this enigmatic object, in particular by obtaining further deep imaging at optical wavelengths.

Erroz-Ferrer et al. (2012) have studied the kinematics of the inner regions of CIG 96 in $\mathrm{H} \alpha$ but no previous study has provided convincing arguments that an external agent can explain both the

\footnotetext{
1 http://amiga.iaa.es 2 This catalog is referred to as K73 in SIMBAD and KIG in NED
databases.
}

Table 1. Parameters of CIG 96 (NGC 864).

\begin{tabular}{cc}
\hline \hline Parameter & Value \\
\hline$\alpha_{(2000)}{ }^{a}$ & $02^{\mathrm{h}} 15^{\mathrm{m}} 27.6^{\mathrm{s}}$ \\
$\delta_{(2000)}{ }^{a}$ & $+6^{\circ} 00^{\prime} 09^{\prime \prime}$ \\
Type $^{b}$ & $\mathrm{SAB}(\mathrm{rs}) \mathrm{c}$ \\
Distance $^{c}$ & $20.3 \mathrm{Mpc}$ \\
$r_{25^{d}}{ }^{c}$ & $2.35^{\prime} / 13.9 \mathrm{kpc}$ \\
Inclination $^{e}$ & $46.59^{\circ}$ \\
$M_{\text {dyn, CIG } 96^{e}}$ & $1.78 \times 10^{11} M_{\odot}$ \\
Position angle $^{e}$ & $20.0^{\circ}$ \\
$A_{\text {int }}(r)^{c}$ & 0.185 \\
$A_{k}(r)^{c}$ & 0.006 \\
$A_{\text {int }}(g)^{c}$ & 0.255 \\
$A_{k}(g)^{c}$ & 0.008 \\
\hline
\end{tabular}

Notes. ${ }^{(a)}$ Leon \& Verdes-Montenegro (2003). ${ }^{(b)}$ de Vaucouleurs et al. (1991). ${ }^{(c)}$ Fernández Lorenzo et al. (2012). Distance computed using $H_{0}=75 \mathrm{~km} \mathrm{~s}^{-1} \mathrm{Mpc}^{-1} . A_{\text {int }}$ and $A_{k}$ represent the internal and $k$ correction extinction terms in SDSS $r$ and $g$ bands. ${ }^{(d)}$ Semi-major axis of the galaxy at the isophotal level $25 \mathrm{mag} \operatorname{arcsec}^{2}$ in the $B$ band (Fernández Lorenzo et al. 2012). ${ }^{(e)}$ This work. Total dynamical mass $M_{\text {dyn }}$ described in Sect. 4.1.

HI and optical features of CIG 96. As a consequence, this raises the question as to whether asymmetries might develop in galaxies free from interactions (Espada et al. 2005, 2011a), motivating the in-depth study of CIG 96.

However, to support any internal agent as the main evolutionary process, it is necessary to first rule out any external influence. Neither tidal features nor gas-rich companions are found in HI maps even for the most asymmetric cases (e.g. Espada et al. 2005, 2011b; Portas et al. 2011; Sengupta et al. 2012) and current shallow optical images are surprisingly symmetric when dust patches are ignored. In the absence of interactions for the last $\sim 2.7$ Gyr (see Sect. 5.1), any lopsided mode would have already dropped (Jog \& Combes 2009). Does this imply that secular evolution processes can lead to asymmetries? Since the early works of Bosma (1978) and Bosma \& Freeman (1993) we know that our understanding of a galaxy may change after performing and comparing deep observations that let us reach very low surface brightness (SB or $\mu$ ) levels of a galaxy and its surroundings. Therefore, this was the natural follow-up for CIG 96. Additionally, as suggested by the N-body simulations of Peñarrubia et al. (2005), the orbital properties of halo substructures are determined by the environment and can survive several gigayears, outliving $\mathrm{HI}$ tidal features. Within the last two decades, a number of works have unveiled many faint structures or companions that remained hidden in shallower observations (e.g. Martínez-Delgado et al. 2008, 2009, 2015; Duc et al. 2015; van Dokkum et al. 2015; Trujillo \& Fliri 2016; Trujillo et al. 2017; Iodice et al. 2017; Bosma 2017, among others).

Espada et al. (2005) also presented the discovery of a close and small companion situated at $15.2^{\prime}(\sim 90 \mathrm{kpc}$, projected distance) to the east of CIG 96. To account for the HI asymmetry, they rule out any encounter with a massive companion as well as any close or parabolic passage of another smaller galaxy. They leave the door open for a parallel passage through the equatorial plane of CIG96 at an intermediate distance, that is, outside the optical disc but within the extended HI disc. Espada et al. (2011a) studied the Kennicutt-Schmidt SF law and efficiency 
Table 2. Data and results of the HI observations with VLA/EVLA.

\begin{tabular}{ccccccc}
\hline \hline Telescope/s & $\begin{array}{c}\text { Velocity range } \\
\left(\mathrm{km} \mathrm{s}^{-1}\right)\end{array}$ & $\begin{array}{c}\text { Integration time } \\
(\mathrm{h})\end{array}$ & $\begin{array}{c}\text { Beam size } \\
\left({ }^{\prime \prime} \times\right)\end{array}$ & $\begin{array}{c}\text { Noise } \\
\left(\mathrm{mJy} \mathrm{beam}^{-1}, 1 \sigma\right)\end{array}$ & $\begin{array}{c}N_{\mathrm{HI}} \text { limit } \\
\left(10^{19} \mathrm{~cm}^{-2}, 5 \sigma\right)\end{array}$ & $\begin{array}{c}M_{\mathrm{HI}} \text { limit } \\
\left(10^{6} M_{\odot}, 5 \sigma\right)\end{array}$ \\
\hline VLA $^{a}$ & $1249.5-1895.2$ & 10 & $27.1 \times 23.6$ & 0.31 & 2.68 & 1.5 \\
EVLA $^{b}$ & $1330-1700$ & 13 & $37.6 \times 20.0$ & 0.84 & 6.19 & 4.1 \\
VLA + EVLA & $1330-1700$ & 19 & $28.0 \times 28.0$ & 0.25 & 1.78 & 1.4 \\
VLA + EVLA + WF $^{c}$ & $1330-1700$ & 19 & $28.0 \times 28.0$ & 0.13 & 0.89 & 0.7 \\
\hline
\end{tabular}

Notes. ${ }^{(a)} 3 \mathrm{~h}$ in D configuration, $7 \mathrm{~h}$ in $\mathrm{C}$ configuration. The original channel width is $10.4 \mathrm{~km} \mathrm{~s}^{-1}(48.8 \mathrm{kHz}) .{ }^{(b)} 3 \mathrm{~h}$ in $\mathrm{D}$ configuration, $10 \mathrm{~h}$ in $\mathrm{C}$ configuration. The original channel width of $3.3 \mathrm{~km} \mathrm{~s}^{-1}$ was smoothed o $10 \mathrm{~km} \mathrm{~s}^{-1}$ (48 kHz) for the current calibration. ${ }^{(c)}$ Wavelet filtering (WF) applied to the VLA + EVLA data.

Table 3. Data of the optical observations.

\begin{tabular}{|c|c|c|c|c|c|c|c|}
\hline $\begin{array}{c}\text { Telescope } \\
\text { (Instrument) }\end{array}$ & Filter & Binning & $\begin{array}{l}\text { Spatial scale } \\
\left({ }^{\prime \prime} \text { pixel }^{-1}\right)\end{array}$ & $\begin{array}{l}\text { Total exp. time } \\
\quad(\# \times \text { time })\end{array}$ & $\begin{array}{l}\text { Field of view }{ }^{c} \\
\left({ }^{\prime} \times / \mathrm{kpc} \times \mathrm{kpc}\right)\end{array}$ & $\begin{array}{c}\text { SB limit } \\
\left(\mathrm{mag} \mathrm{arcsec}^{-2}\right)\end{array}$ & $\begin{array}{c}\text { Seeing } \\
\left({ }^{\prime \prime}\right)\end{array}$ \\
\hline $\begin{array}{l}\text { CAHA2.2m } \\
\text { (CAFOS) }\end{array}$ & Cousins $R$ & $2 \times 2$ & 1.04 & $\begin{array}{c}3 \mathrm{~h} 56 \mathrm{~m} \\
(71 \times 200 \mathrm{~s})\end{array}$ & $\begin{array}{l}12 \times 12 \\
71 \times 71\end{array}$ & 27.5 & 1.59 \\
\hline $\begin{array}{l}\text { CAHA1.23m } \\
\text { (DLR-MKIII) }\end{array}$ & $\begin{array}{c}B, G, R \\
\text { Photographic }^{d}\end{array}$ & $1 \times 1$ & 1.04 & $\begin{array}{c}3 \mathrm{~h} 38 \mathrm{~m} \\
\left(30_{B}, 37_{G}, 42_{R} \times 120 \mathrm{~s}\right)^{e}\end{array}$ & $\begin{array}{l}15 \times 16 \\
88 \times 94\end{array}$ & - & 1.56 \\
\hline $\begin{array}{c}\text { VST } \\
\text { (OmegaCAM) }\end{array}$ & SDSS $r$ & $2 \times 2$ & 0.21 & $\begin{array}{c}\mathrm{h} 10 \mathrm{~m} \\
(122 \times 154 \mathrm{~s})\end{array}$ & $\begin{array}{c}60 \times 60 \\
350 \times 350\end{array}$ & 28.7 & 1.10 \\
\hline
\end{tabular}

Notes. ${ }^{(a)}$ Spatial scale according to the binning used. ${ }^{(b)}$ Total number of exposures $\times$ exposure time of each exposure. ${ }^{(c)}$ The top value of each telescope is the field of view in square arcminutes; the bottom value is the field of view according to the distance to the galaxy (see Table 1). ${ }^{\left({ }^{d}\right)}$ CAHA1.23m images in photographic $B$ and $R$ filters were converted to SDSS $g$ and $r$, respectively (see Sect. 2.4.2). ${ }^{(e)}$ The subindex indicates the filter of each corresponding number of exposures. Regardless of the filter, each one has an exposure time of $120 \mathrm{~s}$.

in the large atomic HI disc of CIG 96, using the VLA observations mentioned in this work (see Sect. 2.1), as well as NUV and FUV observations from GALEX. By comparing the VLA maps and UV images, they found a good spatial correlation between the HI and both NUV and FUV emission, especially outside the inner $1^{\prime}$. Also, the main star-forming regions lie on the enhanced HI emission of two spiral arm-like features that correspond to the HI pseudo-ring. They found that the (atomic) Kennicutt-Schmidt power-law index systematically decreases with the radius. Regarding the star formation efficiency (SFE), they saw that it decreases with radius where the HI component dominates and that there is a break in this correlation at $r=1.5 \times r_{25}$. However, mostly within the HI pseudo-ring structure, that is, between $1.5 \times r_{25}$ and $3.5 \times r_{25}$, SFE remains nearly constant. They concluded that this might be a common characteristic in extended UV disc galaxies and that a non-axisymmetric disc can drive the outer spiral arms, as the morphology of the galaxy allows.

In this work we present new and deep HI and optical data of CIG 96 to study in detail its faint gaseous and stellar components as well as its surroundings in order to reveal any possible causes of its HI asymmetrical distribution and other effects on its evolution. Throughout this study, all mentions to distances between different parts of the galaxy and its surroundings are projected distances unless stated otherwise. Also, we have assumed a cosmology with $H_{0}=75 \mathrm{~km} \mathrm{~s}^{-1} \mathrm{Mpc}^{-1}, \Omega_{\Lambda 0}=0.73$ and $\Omega_{\mathrm{m} 0}=0.27$.

\section{Description of the observations and data processing}

In this section we present all the $\mathrm{HI}$ and optical observations of CIG96 used in this work as well as the reduction and calibration processes we followed to obtain the final images. The most relevant data are summarised in Tables 2 and 3.

\subsection{Hl observations}

In two different epochs, $21 \mathrm{~cm}$ line observations of CIG 96 were made using the NRAO Karl G. Jansky Very Large Array (hereafter VLA or EVLA) observatory. First, two VLA projects AV0276 and AV0282 were performed in July 2004 and July 2005 , respectively. We obtained $3 \mathrm{~h}$ in D-configuration (26 antennas used) and $7 \mathrm{~h}$ in C-configuration (27 antennas used), respectively. Both observing projects had the same set up: 2 IF correlator mode, a bandwidth of $3.125 \mathrm{MHz}$ per IF and a frequency resolution of $48.8 \mathrm{kHz}$ that corresponds to a velocity resolution of $10.4 \mathrm{~km} \mathrm{~s}^{-1}$. Second, the Extended-VLA (EVLA) project 13A-341, fully dedicated to observing CIG 96, was executed during 2013 as follows: $3 \mathrm{~h}$ in March, in D-configuration; $3 \mathrm{~h}$ in May in the hybrid DnC-configuration and $10 \mathrm{~h}$ in July, in C-configuration. In all cases, 27 antennas were used. The set-up of these observations consisted of single IF correlator mode, a bandwidth of $2 \mathrm{MHz}$ and a frequency resolution of $16 \mathrm{kHz}$, equivalent to a velocity resolution of $3.3 \mathrm{~km} \mathrm{~s}^{-1}$ that was smoothed to $10 \mathrm{~km} \mathrm{~s}^{-1}$ for the calculations. These data are summarised in Table 2.

All VLA and EVLA data were fully calibrated and imaged using CASA software package (McMullin et al. 2007) tasks. We used the CLEAN algorithm (Högbom 1974) to produce the final datacube. Each data set or measurement set (MS) was scanned to remove bad data and RFI (radio-frequency interferences). They were separately calibrated in phase, amplitude and bandpass and imaged individually to check their suitability for our aims. We produced a set of two individual datacubes by combining all VLA data and all EVLA data, respectively. We discarded the 
hybrid DnC-configuration data due to the presence of a remarkable amount of RFI, making them too defective for our goals. The VLA data consisted of two individual MSs, one for D-configuration data and one for C-configuration data. EVLA data consisted of thirteen individual MSs: three MSs were obtained in D-configuration and ten MSs in C-configuration. All HI masses in this work have been computed as given by Roberts (1962, 1975):

$$
M_{\mathrm{HI}}\left(M_{\odot}\right)=2.356 \times 10^{5} D^{2} S \Delta V
$$

where $D$ is distance (Mpc) and $S \Delta V$ is the velocity integrated $\mathrm{HI}$ flux $\left(\mathrm{Jy} \mathrm{km} \mathrm{s}^{-1}\right)$.

The column density $N_{\mathrm{HI}}\left(\mathrm{cm}^{-2}\right)$ depends on the brightness temperature $T_{\mathrm{B}}(\mathrm{K})$ integrated over the line width $\mathrm{d} v\left(\mathrm{~km} \mathrm{~s}^{-1}\right)$. In turn, $T_{\mathrm{B}}$ depends on the flux density $S\left(\mathrm{Jy}_{\text {beam }}{ }^{-1}\right)$ and the product of the major and minor axes Maj $\times \min \left(\operatorname{arcsec}^{-2}\right)$. Respectively:

$T_{\mathrm{B}}(\mathrm{K})=6.07 \times 10^{5} \mathrm{~S}(\mathrm{Maj} \times \mathrm{min})^{-1}$

$N_{\mathrm{HI}}\left(\mathrm{cm}^{-2}\right)=1.823 \times 10^{18} \int T_{\mathrm{B}} \mathrm{d} v$

VLA data cube. All VLA data were used to produce a preliminary datacube via imaging using natural weighting. This led to a synthesized beam of $27.11^{\prime \prime} \times 23.60^{\prime \prime}$ and a root mean square $(\mathrm{rms})$ noise level of $0.31 \mathrm{mJy}^{-1}$ beam $^{-1}(1 \sigma)$, reaching a HI column density limit of $N_{\mathrm{HI}}=2.68 \times 10^{19} \mathrm{~cm}^{-2}(5 \sigma)$. Assuming a $\mathrm{HI}$ line width of $10 \mathrm{~km} \mathrm{~s}^{-1}$, the achieved HI mass detection limit is $\sim 1.5 \times 10^{6} M_{\odot}(5 \sigma)$ and a HI column density of $2.7 \times 10^{19} \mathrm{~cm}^{-2}$ $(5 \sigma)$.

EVLA data cube. All EVLA data in C and D configurations were combined and imaged with natural weighting in a preliminary datacube. This datacube had a median rms of 0.84 mJy beam $^{-1}(1 \sigma)$ and a synthesized beam of $37.57^{\prime \prime} \times$ $19.97^{\prime \prime}$. Such beam elongation is due to the short right ascension range in which the observations were taken. With a velocity resolution of $10 \mathrm{~km} \mathrm{~s}^{-1}$, the $\mathrm{HI}$ mass detection limit achieved was of $M_{\mathrm{HI}}=4.1 \times 10^{6} M_{\odot}(5 \sigma)$.

Combined EVLA and VLA data cubes: hereafter the HI cube.

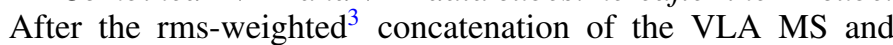
EVLA MS we produced the final datacube of this work (hereafter referred as the HI cube). The corresponding weighting factors applied to the VLA and EVLA data were 10.40 and 1.42, respectively. The HI cube comprises a total of $19 \mathrm{~h}$ on target and has a synthesized beam of $28.16^{\prime \prime} \times 22.72^{\prime \prime}(2.77 \mathrm{kpc} \times 2.24 \mathrm{kpc}$ at a distance of $20.3 \mathrm{Mpc}$ ); it covers a velocity range from $1330 \mathrm{~km} \mathrm{~s}^{-1}$ to $1800 \mathrm{~km} \mathrm{~s}^{-1}$ in 48 channels assuming spectral resolution of $10 \mathrm{~km} \mathrm{~s}^{-1}$. We used the kinematical local standard of rest (LSRK) as the frame of reference for the radio velocities. Also, we worked with a smoothed beam of $28^{\prime \prime} \times 28^{\prime \prime}$ to simplify the physical interpretation of the results and avoid beam effects. The corresponding HI cube yielded a median rms of $0.25 \mathrm{mJy}$ beam $^{-1}(1 \sigma)$ that allowed us to reach a HI mass limit of $M_{\mathrm{HI}}^{\mathrm{lim}} \simeq 1.4 \times 10^{6} M_{\odot}(5 \sigma)$ and a HI column density limit of $N_{\mathrm{HI}} \simeq 1.78 \times 10^{19} \mathrm{~cm}^{-2}(5 \sigma)$. After performing a wavelet filtering (see Sect. 2.2) over the HI cube, we improved these results by a factor of approximately two, reaching a final median rms of $0.126 \mathrm{mJy}$ beam $^{-1}(1 \sigma)$ per channel. The minimum HI mass detected is $M_{\mathrm{HI}}^{\mathrm{lim}}=0.7 \times 10^{6} M_{\odot}(5 \sigma)$, the $\mathrm{HI}$ column density limit is $N_{\mathrm{HI}}=8.9 \times 10^{18} \mathrm{~cm}^{-2}(5 \sigma)$ and the total $\mathrm{HI}$

\footnotetext{
3 Weighting computed as $w(i)=\operatorname{rms}(i)^{-2}$, where $\operatorname{rms}(i)$ stands for the flux density rms of each cube in the same units.
}

mass is $M_{\mathrm{HI}}^{\text {total }}=9.77 \times 10^{9} M_{\odot}(5 \sigma)$. The integrated intensity map, the velocity field and the channel maps are all presented in Sect. 3.

\subsection{Wavelet filtering of the $\mathrm{HI}$ cube}

A robust detection of faint $\mathrm{HI}$ features relies on reaching a column density $\left(N_{\mathrm{HI}}\right)$ that is as low as possible with the best signalto-noise ratio $(\mathrm{S} / \mathrm{N})$. In order to further improve our $N_{\mathrm{HI}}$ limit, we have applied a wavelet filtering to our HI cube which allows to achieve a higher $\mathrm{S} / \mathrm{N}$. An in-depth discussion of the wavelet transform is beyond the scope of this paper but we provide here an explanation of the method used in this work. As explained by Leon et al. (2016), the wavelet transform is a powerful signalprocessing technique that provides a decomposition of the signal into elementary local contributions defined by a scale parameter (Grossmann \& Morlet 1985). The wavelets are the scalar products of shifted and dilated functions of constant shape. The data are unfolded in a space-scale representation that is invariant with respect to dilation of the signal. Such an analysis is particularly suited to studying signals that exhibit space-scale discontinuities and/or hierarchical features, as may be the case for the possible structures located in the outskirts of the HI envelope of CIG 96.

Following the same procedure as Leon et al. (2016), we have used a $B_{3}$-spline scaling function defined by the following convolution matrix $\mathbf{M}$ :

$M=\left[\begin{array}{ccccc}1 / 256 & 1 / 64 & 3 / 128 & 1 / 64 & 1 / 256 \\ 1 / 64 & 1 / 16 & 3 / 32 & 1 / 16 & 1 / 64 \\ 3 / 128 & 3 / 32 & 9 / 64 & 3 / 32 & 3 / 128 \\ 1 / 64 & 1 / 16 & 3 / 32 & 1 / 16 & 1 / 64 \\ 1 / 256 & 1 / 64 & 3 / 128 & 1 / 64 & 1 / 256\end{array}\right]$

Similar to the Ricker function (mexican hat), it has a positive kernel surrounded by a negative annulus and the total integrated area is zero.

We have applied this wavelet over the HI calibrated data via the A trous algorithm (see Bijaoui 1991) as described by Leon et al. (2000). This algorithm creates different filtered wavelet planes according to the scale parameters and a certain threshold level. The scale parameters have received values of $2^{i}$ with $i \in[1,6]$, each defining the $i$ th plane. Each $i$ th raw wavelet plane is defined as the subtraction of two components that, in turn, depend on the $i$ th scale parameter: the zeroth component corresponds to the image plane itself; the rest of the $i$ th components are defined as the result of convolving the $i-1$ th component with the previously defined kernel function. The last plane, namely, the last smoothed plane or LSP (in our case, scale parameter of $2^{6}$ ) does not undergo any convolution; therefore, it is not a wavelet plane itself but the residuals of the last convolution. With the consequent exception of the LSP, each raw plane is filtered above a threshold to construct the $i$ th filtered wavelet plane. For this work, such a threshold was set at $5 \sigma_{i}$, where $\sigma_{i}$ is the rms noise for the $i$ th plane.

The combination of the filtered wavelet planes and the LSP is possible and may cause the rms to change. Since the original image is spread in different spatial scales, a limited combination of the planes implies the recovered flux will be a lower limit to the total emission contribution. Should all planes be combined, the recovery is complete and the total flux is conserved.

After filtering our HI cube, we combined all planes. The resulting rms and, accordingly, the HI column density limit, were improved by a factor of two, as specified in the last paragraph of Sect. 2.1 and summarised in Table 2. 


\subsection{Blanking of the $\mathrm{HI}$ cube}

We separated genuine emission from noise by blanking nonsignal pixels using the following method.

First, we applied a spatial smoothing over the waveletfiltered HI cube by convolving it with a Gaussian kernel four times the size of the adopted synthesized beam, that is, $56^{\prime \prime} \times 56^{\prime \prime}$. The resulting smoothed datacube was only used to create the masks, as described below, and its noise was rms = $0.34 \mathrm{mJy}_{\text {beam }}^{-1}(1 \sigma)$. Second, we created masks for each channel of the smoothed datacube. The shapes of these masks were defined by masking out the pixels with values below a $3.5 \times \mathrm{rms}$ threshold $\left(\sim 1.2 \mathrm{mJy}\right.$ beam $\left.^{-1}\right)$. Finally, the masks from the smoothed datacube were applied to the original datacube (non spatially smoothed) to create the moment maps ${ }^{4}$.

This method mainly has two advantageous consequences: one, the depth and spatial resolution of the original datacube remain unaffected by the masking and two, the threshold limit, for the integration, does not take into account the areas in each channel whose only contribution is noise. In other words, the blanking of the HI cube helps us to remove any remaining effect from the side lobes (either positive or negative) that might mimic nonexistent structures.

\subsection{Optical observations}

In order to obtain deep optical images of the outskirts and close environment of CIG 96, we performed observations in three different observatories. Two datasets were observed with the $2.2 \mathrm{~m}$ and $1.23 \mathrm{~m}$ telescopes, respectively, at $\mathrm{CAHA}^{5}$ observatory in Spain. The first dataset is from CAHA2.2m, a deep image with good seeing in the Cousins $R$ band (see Sect. 2.4.1). The second dataset consists of three images taken with photographic $B, G, R$ bands used to study colour index properties (see Sect. 2.4.2 and all $2.2 \mathrm{~m}$ and $1.23 \mathrm{~m}$ images combined in Fig. 1). The third dataset was obtained with the VLT Survey Telescope $\left(\mathrm{ESO}^{6}\right)$ in Chile (hereafter, VST) and provides a very deep and wide field image to study the surroundings of the galaxy (see image in Fig. 2 and Sect. 2.4.1). The most relevant data are summarised in Table 3.

\subsubsection{CAHA2.2m dataset}

CIG 96 was first observed in the second half of the night of September 11, 2012, with the CAFOS instrument at CAHA2.2m telescope. The CAFOS SITe1d detector has $2048 \times 2048$ pixels with a pixel size of $24 \mu \mathrm{m}$ (spatial scale of $0.53^{\prime \prime}$ pixel $^{-1}$ ), providing an effective circular field of view of $\sim 12^{\prime}$ in diameter.

A total of 71 exposures of $200 \mathrm{~s}$ each build up a total time on source of $3 \mathrm{~h} 56 \mathrm{~m}$. All images were taken in the Cousins $R$ filter, dithered by $\sim 20^{\prime \prime}$ and in $2 \times 2$ binning mode, providing a pixel scale of $1.04^{\prime \prime}$ pixel $^{-1}$. The night conditions were photometric during most of the night, with a median seeing of $1.59^{\prime \prime}$ (seeing ranging from $1.31^{\prime \prime}$ to $\left.1.81^{\prime \prime}\right)$. We used standard reduction and calibration techniques from repipy and LEMON packages ${ }^{7}$ and

\footnotetext{
4 All tasks used to generate the described moment maps are part of the CASA Image Analysis toolkit.

5 Based on observations collected at the Centro Astronómico Hispano Alemán (CAHA) at Calar Alto, operated jointly by the Max-Planck Institut für Astronomie and the Instituto de Astrofísica de Andalucía (CSIC).

6 Based on observations made with ESO Telescopes at the La Silla Paranal Observatory under programme ID 093.B-0894 and 098.B-0775.

7 repipy (https://github.com/javierblasco/repipy) reduction package by J. Blasco-Herrera, LEMON (https://github.com/ vterron/lemon) calibration package by V. Terrón-Salas.
}

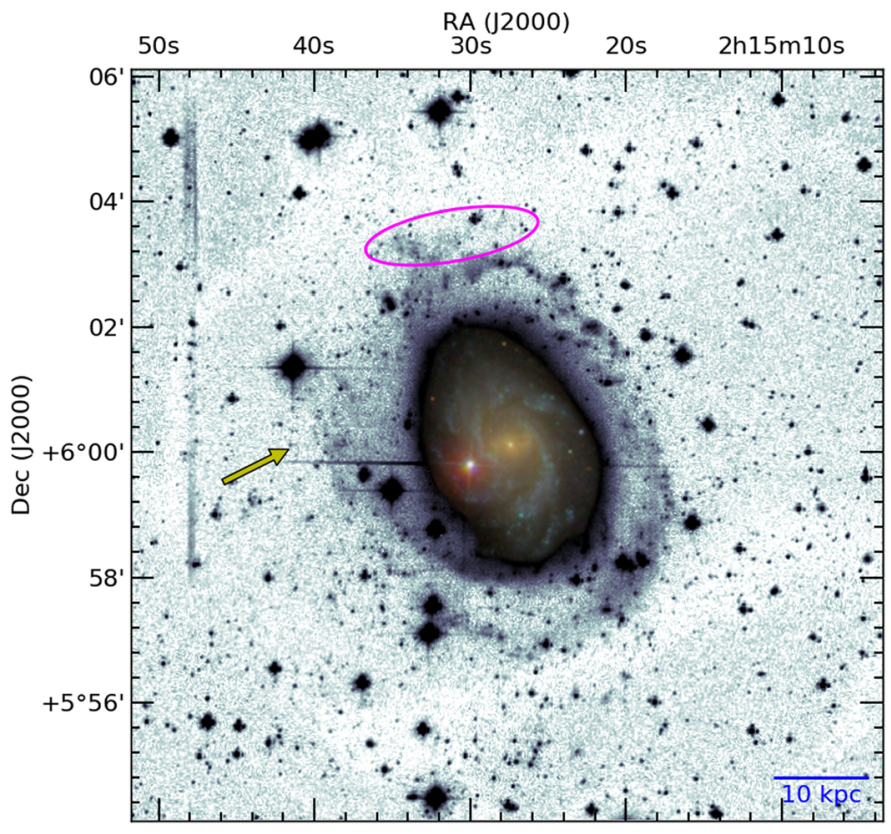

Fig. 1. A $12^{\prime} \times 12^{\prime}$ combined image of the Cousins $R$ image from CAHA2.2m telescope and the three photographic $B, G, R$ images from CAHA $1.23 \mathrm{~m}$ telescope. This particular image is only used to show the outer faint structures of the galaxy (e.g. the pseudo-ring, the northern region in a magenta ellipse or the eastern diffuse emission pointed out by the yellow arrow), not for any physical measurement. The inner coloured area corresponds to an SDSS image of CIG 96 down to $\sim 24 \mathrm{mag} \mathrm{arcsec}^{-2}$ (SDSS $r$ band) and is used as reference.

IRAF. No standard stars were measured in this campaign and so the extinction coefficient was computed by means of nonsaturated stars present within the field of view of our observations. As a consequence, a larger uncertainty is introduced in the photometric calibration. In order to obtain the Zero Point of the night, we computed the Bouguer fit of eight non-saturated stars (visible in all images) and calibrated them with the corresponding data from SDSS (Ahn et al. 2012). Since this dataset was taken using Cousins $R$ filter, all fluxes were converted from SDSS magnitudes system to Cousins $R$ using the transformation by Lupton (2005), derived by matching photometry data from SDSS Data Release 4 (DR4) to Peter Stetson's published photometry for stars:

$R_{r i}=r-0.2936 *(r-i)-0.1439$

in magnitudes, where $r$ and $i$ are the magnitudes in the SDSS $r$ and SDSS $i$ filters, respectively. The median Zero Point of the night (Cousins $R$ filter) is $24.28 \pm 0.12 \mathrm{mag}$. We calculated the SB of the image by setting 40 square boxes of $20^{\prime \prime} \times 20^{\prime \prime}$ size in the southern, western and northern areas of the image. The eastern side of the CAHA $2.2 \mathrm{~m}$ image is heavily contaminated by a star so we did not take into account any SB measurements of that side. There is a slightly uneven distribution of the light between the western side (median $\mu_{\text {Cous } R}=27.5 \mathrm{mag} \operatorname{arcsec}{ }^{-2}$ ) and the northern and southern sides $\left(\mu_{\text {Cous } R}=28 \mathrm{mag} \mathrm{arcsec}^{-2}\right)$. We cannot confirm whether the $0.5 \mathrm{mag} \operatorname{arcsec}^{-2}$ difference comes from the residuals of the flat-fielding or from reflected light and the small field of view of the image prevents selecting a SB value over the rest so we set the SB limit of the image as the lowest value, $\mu_{\text {Cous } R}=27.5 \mathrm{mag} \mathrm{arcsec}^{-2}$ (approximately $\mu_{\text {SDSS } r}=28.0 \mathrm{mag} \operatorname{arcsec}^{-2}$ ). 


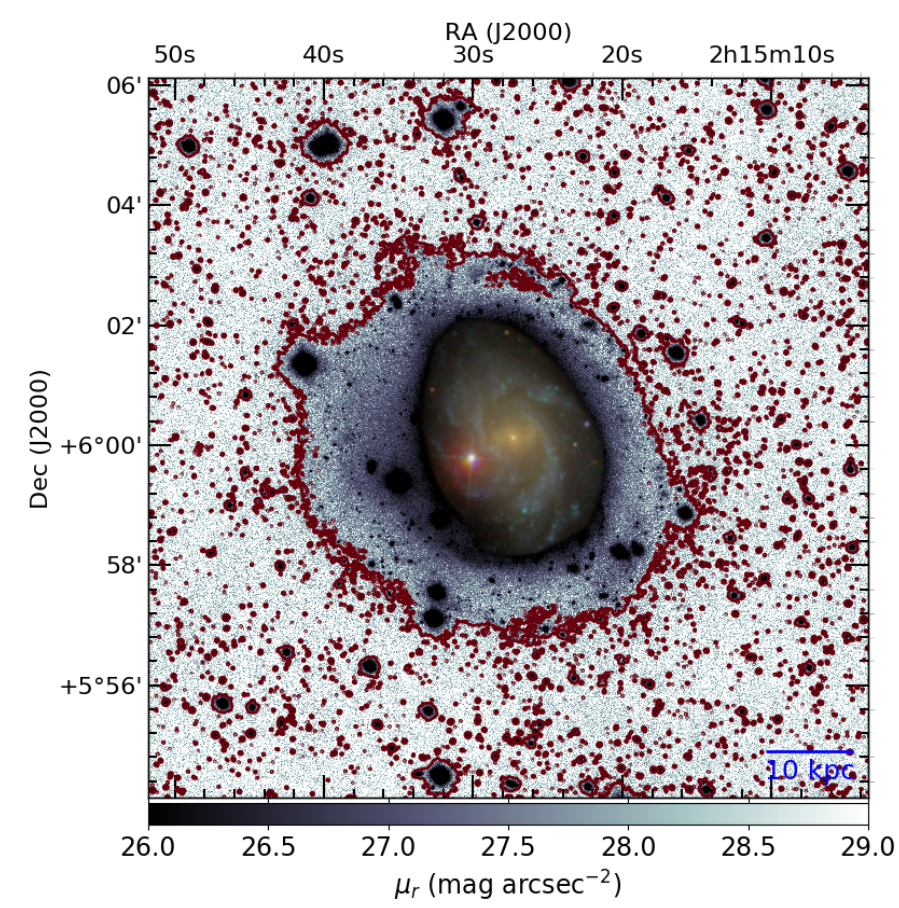

Fig. 2. A $12^{\prime} \times 12^{\prime}$ detail of the VST optical image of CIG 96 with the SDSS colour image down to $\sim 24 \mathrm{mag} \operatorname{arcsec}^{-2}$ (SDSS $r$ ). The red contour is set on $26.5 \mathrm{mag} \operatorname{arcsec}^{-2}$ (SDSS $r$ ), to point out the faintest SB level of the pseudo-ring.

\subsubsection{CAHA1.23m dataset}

CIG 96 was observed for a second time on the night of December 8, 2012 with the DLR-MKIII instrument at the CAHA $1.23 \mathrm{~m}$ telescope. The camera is equipped with an e2v CCD231-84NIMO-BI-DD sensor $\left(4 \mathrm{k} \times 4 \mathrm{k}\right.$ pixels, $\left.15 \mu \mathrm{m} \mathrm{pixel}^{-1}\right)$. The original field of view is $21.5^{\prime} \times 21.5^{\prime}$ but the observations were cropped down to the central $15^{\prime} \times 16^{\prime}$.

In this case, we used three different filters: photographic $B$, $G$ and $R$ (different from Johnson-Cousins) for which a total of 30,37 and 42 exposures of $120 \mathrm{~s}$ each were taken, respectively, in $1 \times 1$ binning mode. The night conditions were stable for the most part of the night and all filters present a median seeing of $1.56^{\prime \prime}$ (seeing range from $1.48^{\prime \prime}$ to $1.61^{\prime \prime}$ ). The total integration time was $3 \mathrm{~h} 38 \mathrm{~m}$.

As with the previous dataset, standard reduction was applied to all the images in each filter separately. However, they were divided by a blank field. It was obtained from an adjacent galaxyfree field and corrected for bias and regular flat field too, so the remaining image would not show any residual gradient. Dividing the images by this blank field allows large-scale structures to be removed. We used the SDSS tabulated fluxes from several stars to calibrate the images via the following relation between SDSS and photographic filters: $B(3900-5100 \AA)$ would correspond to SDSS $g$ and $R(5800-7000 \AA)$ to $\operatorname{SDSS} r$. However, $G(4900-5800 \AA$ ) would lie right between SDSS $g$ and $r$ bands. For the conversion of $G$ band to SDSS, we considered different scenarios in which the emission was split between SDSS $g$ and $r$ bands but it has not been used further in this work. Hereafter we focus on the empirical relations that we calculated for $R$ and $B$ bands with respect to SDSS $r$ and $g$. The initial relations between the corresponding magnitudes (not corrected from extinction) are:

$m_{r_{\text {SDSS }}}^{+ \text {ext }}=1.01 * m_{R_{\text {phot }}}-9.83 \pm 0.15$ and

$m_{g_{\mathrm{SDSS}}}^{+\mathrm{ext}}=0.99 * m_{B_{\mathrm{phot}}}-9.70 \pm 0.33$.

Internal extinction and $k$-correction were applied to the fluxes in both $g$ and $r$ bands. We used the extinction laws by Savage \& Mathis (1979; in agreement with Fitzpatrick 1999) where $A(B)=4.10 \times E_{B-V}$; the internal extinction and $k$ correction in the $B$ band for CIG 96 are $A_{\text {int }}(B)=0.276$ and $A_{k}(B)=0.009$, respectively (Fernández Lorenzo et al. 2012); the extinction-reddening relations for the SDSS bands are $A_{x}(g)=3.793 \times E_{B-V}$ and $A_{x}(r)=2.751 \times E_{B-V}$ (Stoughton et al. 2002).

These relations yield the following internal and $k$-correction values for each band: $A_{\text {int }}(g)=0.255, A_{\text {int }}(r)=0.185, A_{k}(g)=$ 0.008 and $A_{k}(r)=0.006$.

Hence, the final empirical extinction-corrected equations that convert photographic $B$ and $R$ bands to SDSS $g$ and $\operatorname{SDSS} r$ bands are:

$m_{r_{\mathrm{SDSS}}}=1.01 * m_{R_{\mathrm{phot}}}-10.02 \pm 0.15$

and:

$m_{g_{\mathrm{SDSS}}}=0.99 * m_{B_{\text {phot }}}-9.96 \pm 0.33$.

Finally, the images were average stacked applying an outlierrejection algorithm.

With the two images from $B$ and $R$ bands already calibrated to SDSS $g$ and SDSS $r$ bands respectively, we built a $g-r$ image with the aim of studying the colour distribution in the most interesting regions of the galaxy (see Sect. 4.3).

In Fig. 1 we show the result of combining the reduced CAHA2.2m image (Cousins $R$ band) and the three reduced CAHA1.23m images (photographic filters). The lower resolution of these images (compared to the better resolution of VST, see Sect. 2.4.3) provides a more clear visualization of the external structures of CIG 96, especially the faint structure in the $\mathrm{N}$ and the very diffuse $\mathrm{E}$ side of the pseudo-ring, indicated in the image. However, we cannot calibrate them all to a common band, so this image must be taken only as an illustrative view of the galaxy.

\subsubsection{VST dataset}

In order to study the larger-scale structure surrounding CIG 96, we also observed the galaxy with OmegaCAM at the VST (runID: 098.B-0775(A)). This instrument has a field of view of 1 square degree sampled with a $32-\mathrm{CCD}, 16 \mathrm{k} \times 16 \mathrm{k}$ detector mosaic at $0.21^{\prime \prime}$ pixel $^{-1}$.

The 32 CCDs have intermediate spaces between the different chips in the vertical direction $(5.64 \mathrm{~mm}$ top and bottom gaps; $0.82 \mathrm{~mm}$ central gap) and in the horizontal direction $(1.5 \mathrm{~mm}$ gap). Also, at the time these observations were designed, the user manual accounted for cross talk between CCDs 93-96 at $<0.4 \%$ level (slightly above our aim of $0.35 \%$ ). Further discussion with the telescope staff alerted to irregular gain variations in CCDs 82, 87 and 88. In order to avoid these CCDs as much as possible and guarantee a homogeneous coverage of the gaps, we initially designed a manual diagonal dithering pattern for the pilot observations exposures to sample the galaxy and its surroundings. With it, the 49 different offset positions of the galaxy (7 pointings with 7 offset positions each) were placed along a diagonal oriented from the southeast (SE) to the northwest (NW) of the chip, always leaving at least $1^{\prime}$ (both in RA and Dec) with the edge of the CCDs. After the pilot observations, we concluded 
that the previous diagonal dithering would not significantly differ from the already existing modes (JITTER and DITHER, since STARE was not useful for our aims) so we designed a new manual dithering pattern that would make a total of seven pointings, six of them to the corresponding apexes of a slightly irregular hexagon-shaped pattern plus one more central pointing.

A total of eight observing blocks (OBs) of $1 \mathrm{~h}$ each were dedicated to observing CIG 96. From these, 7 OBs had 16 exposures and $1 \mathrm{OB}$ had 10 exposures, making a total of 122 exposures of $154 \mathrm{~s}$ each. The 8 OBs were carried out on the nights of October 6, 9 and 20, November 1 and 2 and December 2, 3 and 20, all in 2016. The total time spent on source was $5 \mathrm{~h} 10 \mathrm{~m}$. All observations were done under the following conditions: photometric sky transparency, maximum seeing of $2.0^{\prime \prime}$, airmasses below 2.0 $\left(>63.4^{\circ}\right)$, with an angular distance to the Moon of at least $60^{\circ}$ and its maximum illumination at $30 \%$. We used a modified version (Venhola et al. 2017) of Astro-WISE pipeline (McFarland et al. 2013) to reduce and calibrate these data. The SB of the image was calculated as the median of the SB values computed in $\sim 60$ square boxes of $20^{\prime \prime} \times 20^{\prime \prime}$ spread in the central $40^{\prime} \times 40^{\prime}$ of the image and avoiding stars. For this we used Eq. (10), in which the second term corresponds to the conversion from pixel $^{-2}$ to $\operatorname{arcsec}^{-2}$ :

$$
\begin{aligned}
\mu_{\text {SDSS } r} & =-2.5 * \log \left(F_{\text {SDSS } r}\right)-2.5 * \log \left(0.21^{2}\right) \\
& =-2.5 * \log \left(F_{\text {SDSS } r}\right)+3.3889 .
\end{aligned}
$$

Figure 2 shows an SDSS colour image of CIG 96 on top of a subset of the VST image. Additionally, the faint SB reached with this image allowed us to detect Galactic cirrus around the galaxy (see Sect. 4.6).

\subsection{Planck and WISE images}

In order to inspect the cirrus around CIG 96 (see Sect. 4.6), we used images from the HFI camera of the Planck satellite at $857 \mathrm{GHz} / 350 \mu \mathrm{m}$ band (Planck Collaboration I 2014). Also, we have used a WISE band 3 image $(12 \mu \mathrm{m})$ since this band that traces hot dust and shows good correlation with the cirrus emission (Miville-Deschênes et al. 2016). Throughout this work, we will refer to these images as Planck857 and WISE3, respectively. Planck857 images were obtained from SkyView online tool (McGlynn \& Scollick 1994) while the WISE3 image was obtained from the IRSA, NASA/IPAC archive and was reprocessed to improve the flat fielding and remove the stars.

\section{3. $\mathrm{HI}$ results}

\subsection{Integrated emission and asymmetry level}

To calculate the total spectrum, we integrated the emission of each channel of the HI cube. Then, as discussed by Fouque et al. (1990), we computed the central velocity of the galaxy $V_{c e n}$ as the average between the lowest and highest velocities measured at a width (or flux level) of the $20 \%$ of the highest flux peak in the integrated spectrum (abbreviated W20, name varies depending on the percentage used). The error can be estimated as: $\Delta V=4 \frac{\sqrt{\delta v(W 20-W 50) / 2}}{S / N_{\text {peak }}}$ where $\delta v$ is the spectral resolution of the cube, $(W 20-W 50) / 2$ represents the steepness of the edges of the HI profile at $20 \%$ and $50 \%$ of the maximum flux, and $\mathrm{S} / \mathrm{N}_{\text {peak }}$ is the $\mathrm{S} / \mathrm{N}$ of the maximum flux peak. Taking these

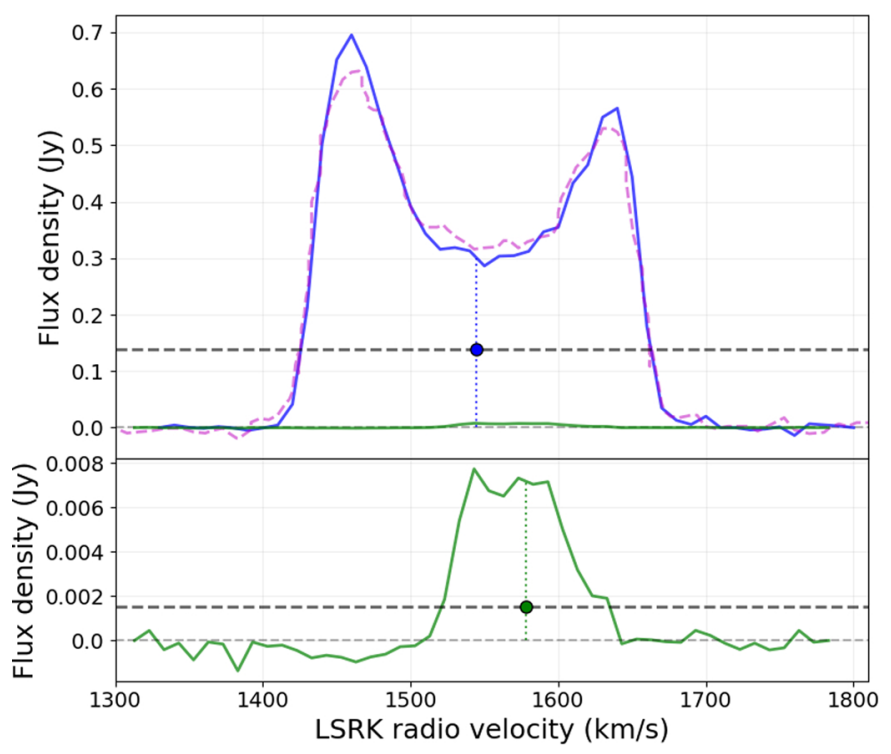

Fig. 3. Top panel: integrated profile of CIG 96, calculated from the EVLA and VLA combined HI cube (blue solid line), integrated spectrum of CIG 96 (LSRK) obtained by Haynes et al. (1998) at Green Bank $43 \mathrm{~m}$ (heliocentric) (pink dashed line). Our integrated spectrum shows a central velocity that is lower than the Green Bank spectrum, therefore in order to match and facilitate the comparison between the two, we have shifted the latter by $-17 \mathrm{~km} \mathrm{~s}^{-1}$. The green solid line is the integrated profile of the closest companion of CIG 96: NGC 864 COM01. The horizontal green dashed line sets the width at $20 \%$ of the highest flux peak $(W 20)$ for the central radio velocity computation, shown as a blue dot $\left(V_{\mathrm{LSRK}}(\mathrm{CIG} 96)=1544.15 \mathrm{~km} \mathrm{~s}^{-1}\right)$. The vertical blue dotted line defines the two halves of the spectrum for the asymmetry parameter calculation. Bottom panel: integrated HI profile of the companion NGC 864 COM01 with a rescaled flux density for an easier visualization. The green dot sets the central velocity of this galaxy $\left(V_{\mathrm{LSRK}}(\right.$ companion $\left.)=1577.90 \mathrm{~km} \mathrm{~s}^{-1}\right)$.

into account, the $W 20$ central radio velocity of our $\mathrm{HI}$ cube is $V_{\text {LSRK }}\left(\right.$ CIG 96) $=1544.15 \pm 0.23 \mathrm{~km} \mathrm{~s}^{-1}$.

We find a difference of approximately $10 \mathrm{~km} \mathrm{~s}^{-1}$ between our result for the central velocity of CIG 96 and those calculated from single-dish data by Espada et al. (2005; same method as in this work) and Haynes et al. (1998), 1561.6 and $1562 \pm 1 \mathrm{~km} \mathrm{~s}^{-1}$, respectively, both in heliocentric frame of reference, that is, approximately 1553 and $1554 \mathrm{~km} \mathrm{~s}^{-1}$ when converted to LSRK, as is ours. Kerr \& Lynden-Bell (1986) also provide a LSRK velocity of $1553 \pm 1 \mathrm{~km} \mathrm{~s}^{-1}$, showing the same shift with respect to our result. To identify the reason for this apparent inconsistency, we recalculated the central velocity of our $\mathrm{HI}$ cube and the one published by Espada et al. (2005) in different standards of rest and in the two optical and radio velocity conventions. In all cases, the differences remained within a few $\mathrm{km} \mathrm{s}^{-1}$, i.e., no change in the standard of rest or velocity convention would account for such a shift. The calibration process was also revised and the correct rest frequency for the HI line was confirmed, leaving us with the only hypothesis of an undetected error in the raw data or the calibration process. Taking this into account, we conclude this difference may be assumed, not to affect the interpretation of the data in any case since it is a small shift compared to the width of the profile.

CIG 96 has a close companion: NGC 864 COM01 (hereafter also referred to as the companion), detected in $\mathrm{HI}$ by Espada et al. (2005). We determine a $W 20$ central radio velocity of $V_{\mathrm{LSRK}}($ companion $)=1577.90 \pm 2.62 \mathrm{~km} \mathrm{~s}^{-1}$. The HI and 
optical properties of this galaxy, as well as its implication in the isolation of CIG 96, are discussed further in Sect. 3.3, 4.5 and 5.1 .

In Fig. 3 we compare the integrated emission spectra derived from our HI cube for CIG 96 and its companion with the one obtained by Haynes et al. (1998) using data from Green Bank $43 \mathrm{~m}$ single dish telescope, and still in the heliocentric system of reference. For a better comparison between the two spectra, we have shifted the latter by $-17 \mathrm{~km} \mathrm{~s}^{-1}$. The perfect match between them strongly suggests that our HI cube has a velocity shift of $-10 \mathrm{~km} \mathrm{~s}^{-1}$, after converting all velocities to LSRK.

In order to estimate the HI asymmetry level of a galaxy, quantified as $A_{\text {flux ratio }}$ (e.g. Haynes et al. 1998; Kornreich et al. 2001; Espada et al. 2011b) we also use the HI integrated spectrum. $A_{\text {flux ratio }}$ is an areal asymmetry parameter defined as the emission ratio set between the two regions of the spectrum defined with respect to the central velocity and its lowest and highest velocity. While it provides a simple quantification of the gas distribution in the two halves of the galaxy, this global parameter does not give spatial information of any possible asymmetry.

We calculated the sources of the uncertainties of this parameter as described by Espada et al. (2011b), obtaining $A_{\text {flux ratio }}=1.16 \pm 0.01$, that is, $16 \pm 1 \%$, in full concordance with Espada et al. (2005).

\subsection{Channel maps}

The channel maps allow to inspect every channel of the HI data cube. Each one corresponds to a different velocity allowing us to trace any structures that might be connected to the gaseous envelope of the galaxy. In Fig. 4 we show a subset of the channel maps of the wavelet filtered HI cube on top of the CAHA $1.23 \mathrm{~m}$ optical image (band $R$ ) of CIG 96. This image corresponds to the central $25^{\prime} \times 25^{\prime}$ of the primary beam and to the channels with emission, that is, from 1380 to $1690 \mathrm{~km} \mathrm{~s}^{-1}$ (channels 6 to 37 , respectively) where the channel width is $10 \mathrm{~km} \mathrm{~s}^{-1}$. The systemic velocity of the galaxy $\left(V_{\mathrm{LSRK}}(\mathrm{CIG} 96)=1544.15 \mathrm{~km} \mathrm{~s}^{-1}\right.$, see Sect. 3.1) corresponds to channel 23 and the approaching and receding sides of the galaxy extend approximately 135 and $145 \mathrm{~km} \mathrm{~s}^{-1}$, respectively. The synthesized beam is $28^{\prime \prime} \times 28^{\prime \prime}$, the rms is $0.126 \mathrm{mJy}^{-1}$ beam $^{-1}$ and the column density reached is $N_{\mathrm{HI}}(5 \sigma)=8.9 \times 10^{18} \mathrm{~cm}^{-2}$.

The HI distribution is more symmetrical in the central channels $\left(\sim 1500-1600 \mathrm{~km} \mathrm{~s}^{-1}\right)$ than in those with velocity differences of $\Delta V \geqslant 60 \mathrm{~km} \mathrm{~s}^{-1}$ with respect to the central velocity. In the latter, the approaching side shows that the HI has a uniform distribution over a larger area in the southwest (SW) than in the receding side, where the distribution is more narrow and oriented towards the northeast (NE). The HI extension also differs, reaching $\sim 7.9^{\prime}(\sim 47 \mathrm{kpc})$ towards the SW and $\sim 9.3^{\prime}(\sim 55 \mathrm{kpc})$ towards the NE. Also, the receding NE side is less massive, as reflected in the asymmetrical shape of the integrated spectrum (Sect. 3.1). In both the approaching and receding sides, the $\mathrm{HI}$ is extended beyond $4 \times r_{25}$ of the optical extension. From 1630 to $1670 \mathrm{~km} \mathrm{~s}^{-1}$ (channels 31 to 35 ), there is a change in the orientation of the $\mathrm{HI}$, especially visible in column densities below $1.0 \times 10^{19} \mathrm{~cm}^{-2}$ (outer contours of Fig. 4 and moment maps shown in Sect. 3.4).

Focusing on the outermost regions, we note two previously undetected features:

- First, from 1480 to $1550 \mathrm{~km} \mathrm{~s}^{-1}$ (channels 16 to 23), we notice a clumpy structure to the NW of the galaxy $(\alpha=$ $\left.02^{\mathrm{h}} 15^{\mathrm{m}} 05.9^{\mathrm{s}}, \delta=6^{\circ} 03^{\prime} 03^{\prime \prime}\right)$, with an approximate size of $\sim 21 \mathrm{kpc}(\sim 3.5 \mathrm{arcmin}$, measured from channels 17 to 22$)$, a column density of approximately $N_{\mathrm{HI}}^{\mathrm{NW}} \simeq 6.5 \times 10^{19} \mathrm{~cm}^{-2}$ and a total HI mass of $M_{\mathrm{HI}}^{\mathrm{NW} \text { feat. }} \simeq 3.1 \times 10^{6} M_{\odot}$. We refer to this as the NW HI feature and it is indicated with green marks in Fig. 4.

- Second, from 1600 to $1640 \mathrm{~km} \mathrm{~s}^{-1}$ (channels 28 to 32), a structure shows up to the SE of the galaxy $(\alpha=$ $\left.02^{\mathrm{h}} 15^{\mathrm{m}} 41.0^{\mathrm{s}}, \delta=5^{\circ} 55^{\prime} 31^{\prime \prime}\right)$, within a square region of approximately $8.8 \times 8.8 \mathrm{kpc}\left(\sim 90^{\prime \prime} \times 90^{\prime \prime}\right)$ size, a column density of approximately $N_{\mathrm{HI}}^{\mathrm{NW}} \simeq 4.9 \times 10^{19} \mathrm{~cm}^{-2}$ and a total HI mass of $M_{\mathrm{HI}}^{\mathrm{SE} \text { feat. }} \simeq 1.6 \times 10^{6} M_{\odot}$. We refer to this as the SE HI feature and it is indicated with magenta marks in Fig. 4.

These structures are discussed further in Sect. 5.2.

\subsection{NGC 864 COM01, the HI rich companion of CIG 96}

As described by Espada et al. (2005) and introduced in Sect. 3.1, CIG 96 has a small companion located at 15.2' ( $90 \mathrm{kpc})$ to the east with a $B$ magnitude of $m_{B}=16.38$ mag. It shows emission throughout 11 channels (from 1540 to $1650 \mathrm{~km} \mathrm{~s}^{-1}$ ). Its central LSRK velocity is of $V_{\mathrm{LSRK}}=1577.90 \mathrm{~km} \mathrm{~s}^{-1}$ and a total HI mass of $M_{\mathrm{HI}}=5.1 \times 10^{6} M_{\odot}$. The HI image of this galaxy is shown in Figs. 5 and 7. Both CIG 96 and its companion share a similar orientation of their minor axis. However, they show different kinematical orientation, that is, the companion is counterrotating with respect to CIG 96, and we do not find any signs of tidal features between them. The galaxy is studied further in Sect. 4.5.

\subsection{Moment maps and position-velocity profiles}

The integration of the flux density $S$ (or zeroth moment) is carried out from channel $6\left(1380 \mathrm{~km} \mathrm{~s}^{-1}\right)$ to channel 38 $\left(1700 \mathrm{~km} \mathrm{~s}^{-1}\right)$, i.e. one additional channel beyond the HI emission. The velocity field (or first moment) is the intensityweighted velocity of the spectral line, i.e., a measure for the mean velocity of the gas. The zeroth moment is shown in Figs. 5 and 6 . The HI extends beyond $4 \times r_{25}$, that is, approximately up to $50 \mathrm{kpc}\left(8.5^{\prime}\right)$, reaching an integrated column density of $N_{\mathrm{HI}}(5 \sigma)=1.2 \times 10^{20} \mathrm{~cm}^{-2}$ with a beam size of $28^{\prime \prime} \times 28^{\prime \prime}$. As a comparison, in Fig. 5 we indicate with a black line the approximate $N_{\mathrm{HI}}(5 \sigma)=8.7 \times 10^{20} \mathrm{~cm}^{-2}$ column density reached by Espada et al. (2011a) with a beam size of $16.9^{\prime \prime} \times 15.6^{\prime \prime}$. Quantitatively, the current observations are roughly seven times deeper than the previous ones.

The first moment is shown in Fig. 7. It allows the estimation of the position angle (from now on, PA) of the major and minor kinematical axes of the galaxy, indicated by the two black lines at $\mathrm{PA}=20^{\circ}$ and $\mathrm{PA}=110^{\circ}$, respectively.

We have performed the position-velocity $(\mathrm{P} / \mathrm{V})$ profiles over the HI cube along the major and minor axes, as shown in Fig. 8. The emission located at the largest radius in the SW region (indicated with a cyan arrow in the profile over the major axis, Fig. 8, top panel) was already detected by Espada et al. (2005). It is visible in the channel maps at $1450-1470 \mathrm{~km} \mathrm{~s}^{-1}$ (channels 13-15) and it shows a drop in velocity of about $30-40 \mathrm{~km} \mathrm{~s}^{-1}$ with respect to inner parts of the galaxy. Both its extension and velocity drop are in agreement with the previous work. The interruption in the emission to the NE is due to a $\sim 3 \times 3 \mathrm{kpc}^{2}$ region $\left(\sim 30^{\prime \prime} \times 30^{\prime \prime}\right)$ with low HI emission. It is visible in the zeroth moment map $\left(\mathrm{RA}=2^{\mathrm{h}} 15^{\mathrm{m}} 34.935^{\mathrm{h}}\right.$, Dec $\left.=6^{\circ} 04^{\prime} 33.17^{\prime \prime}\right)$ as well as in the channel maps at $1630-1640 \mathrm{~km} \mathrm{~s}^{-1}$ (channels 31 and 32).

The $\mathrm{P} / \mathrm{V}$ profile over the minor axis cuts through part of the NW HI feature (indicated with a red arrow, Fig. 8, bottom panel), the clumpy HI structure mentioned in Sect. 3.2. This feature 


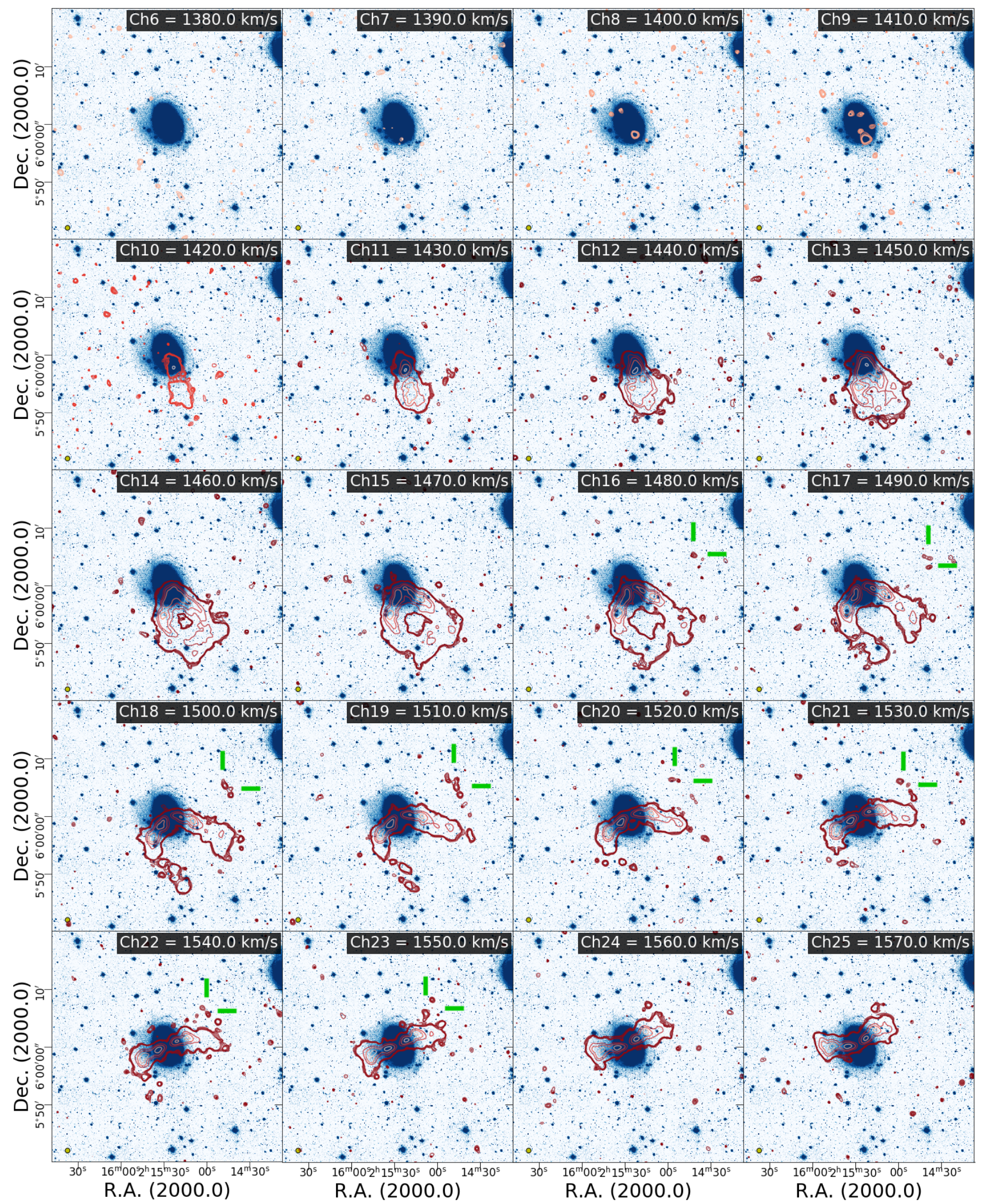

Fig. 4. Channel maps of the wavelet filtered HI cube superimposed on the VST optical image of CIG 96 . The field of view is approximately $25^{\prime} \times 25^{\prime}$ $(147 \times 147 \mathrm{kpc})$. Foreground: red contours correspond to 3.4, 3.9, 4.5, 5.1, 5.6, 28.1, 56.2, 112.5 and $224.9 \sigma$ levels $\left(\mathrm{rms}=0.126 \mathrm{mJy} \mathrm{beam}^{-1}, 1 \sigma\right)$ or the equivalent $\mathrm{HI}$ column densities of $0.6,0.7,0.8,0.9,1.0,5.0,10.0,20.0,40.0 \times 10^{19} \mathrm{~cm}^{-2}$, respectively. Green and magenta marks indicate the NW and SE HI features, respectively. The synthesized beam of $28^{\prime \prime} \times 28^{\prime \prime}$ is shown in the bottom left corner as a yellow circle. Background: VST image of CIG 96. We display a SB range of $\mu_{r \text { SDSS }}=26.0-28.4 \mathrm{mag} \mathrm{arcsec}^{-2}$ to enhance the outskirts of the galaxy while brighter inner structures are not shown. 


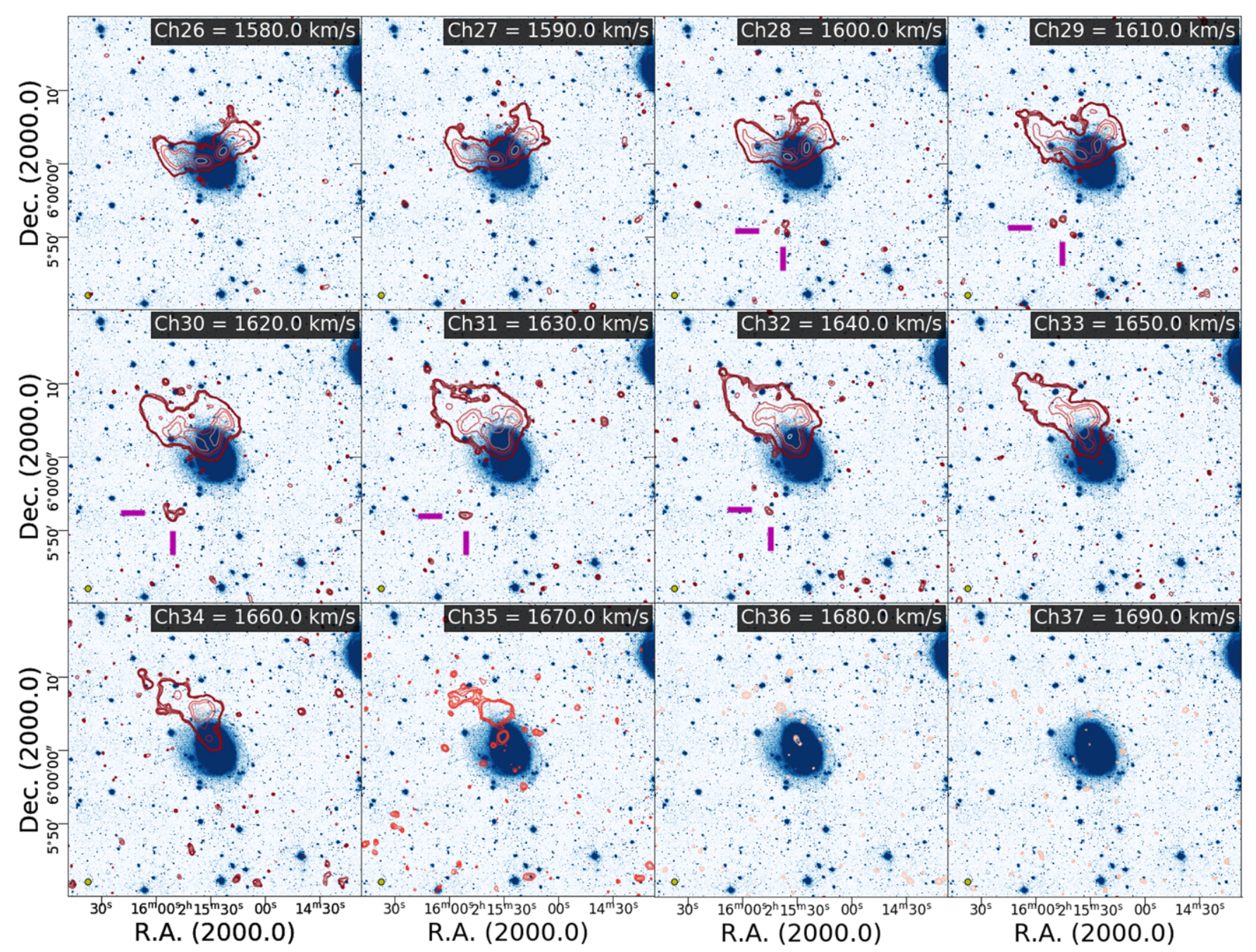

Fig. 4. continued.

shows a velocity gradient of $\sim 70 \mathrm{~km} \mathrm{~s}^{-1}$ (approximately from 1480 to $1550 \mathrm{~km} \mathrm{~s}^{-1}$ ) and it seems to connect with the galaxy in the channels around its central velocity (channels 23 to 25). Also, the central part of the galaxy shows emission in a wide range of velocities with respect to the central velocity. We discuss this effect further in Sect. 5.2.

\section{Optical data results}

\subsection{Surface brightness limit, dynamical masses and optical features}

The images from CAHA2.2m and CAHA1.23m telescopes have a field of view of $12^{\prime} \times 12^{\prime}$, i.e. approximately $71 \times 71 \mathrm{kpc}$ (see Fig. 1), while the VST covers $1^{\circ} \times 1^{\circ}$, that is, approximately a $350 \times 350 \mathrm{kpc}$ field centred on the galaxy. The limiting SB reached is deeper than any other previously published, in particular with the VST image $\left(\mu_{r}\right.$ SDSS $($ VST $)=28.7 \mathrm{mag} \mathrm{arcsec}^{-2}$, see Fig. 2) that reveals unprecedented detail of the extension, boundaries and structures of the external and faint pseudoring of CIG96 as well as its connection to the inner parts of the galaxy. The VST image also shows signs of Galactic cirrus (see Sect. 4.6) so we set our reliable detection limit in $\mu_{r \text { SDSS }}(\mathrm{VST})=28.4 \mathrm{mag} \operatorname{arcsec}^{-2}$, just above the level where they start to become visible.
The total dynamical mass of CIG 96 is $M_{\text {dyn, CIG } 96}=1.78 \times$ $10^{11} M_{\odot}$, following the calculation described by Courteau et al. (2014). It was estimated taking into account the inclination $(i$, in degrees, indicated in Table 1), the radius of the galaxy along the major axis $(R$, in kpc) as well as the rotation velocity $(V$, in $\mathrm{km} \mathrm{s}^{-1}$ ). Both $R$ and $V$ are extracted from the HI data: $R$ of $6^{\prime}(35.43 \mathrm{kpc})$ from the rotation curve of the major axis (see Sect. 3.4) and $V$ via measuring the velocity difference at such radius with respect the central velocity of the galaxy, resulting in $125 \mathrm{~km} \mathrm{~s}^{-1}$. The same calculation was made for the companion. We obtained a $\mathrm{P} / \mathrm{V}$ cut of the galaxy along a PA of $35^{\circ}$ to measure the peak $R$ and $V$, resulting in $35^{\prime \prime}(3.44 \mathrm{kpc})$. However, with the current data we do not observe a turn over in the rotation curve so the mass calculation at this radius must be taken as a lower limit. We also assumed an inclination of $90^{\circ}$ since it might be an edge-on galaxy (discussed further in Sect. 4.5). The velocity extent measured at a $35^{\prime \prime}$ radius is of $60 \mathrm{~km} \mathrm{~s}^{-1}$. The dynamical mass of the companion is of $M_{\text {dyn, comp }}=2.88 \times 10^{9} M_{\odot}$. Hence, the dynamical mass relation between the host galaxy and its companion is approximately of $M_{\text {dyn, CIG } 96} / M_{\text {dyn, comp }} \simeq 62$. The case of CIG 96 can be considered similar to the one of the MW-mass galaxy M94 that, after a deep search performed as part of the recent work by Smercina et al. (2018), only shows two satellites. 


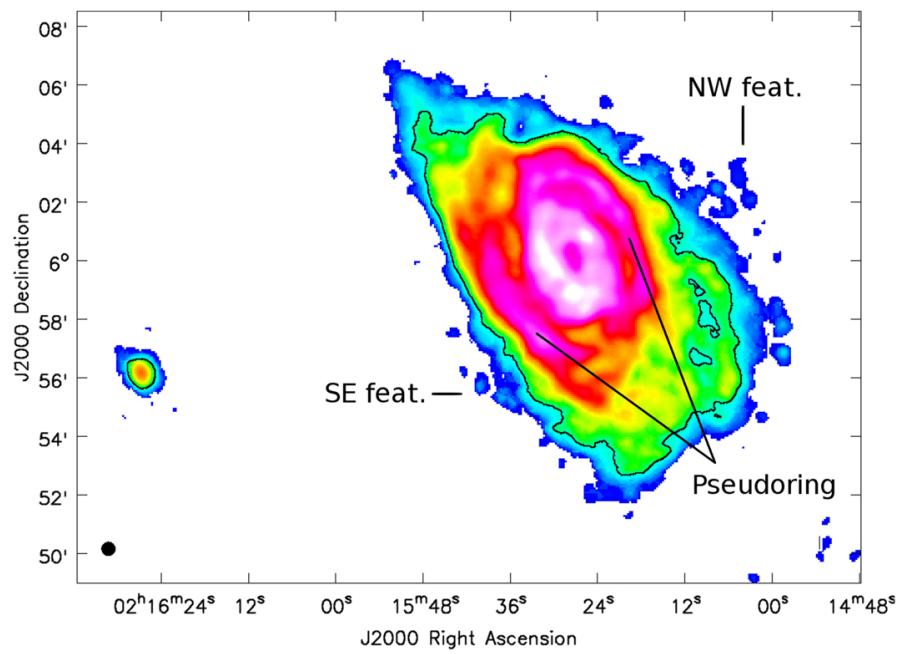

Fig. 5. HI integrated intensity map of CIG 96 and its companion after a $3.5 \sigma$ blanking (see Sect. 2.3). We identify the NW and SE HI features mentioned in Sect. 3.2 as well as the HI emission of the pseudoring. The black contour represents the column density of $N_{\mathrm{HI}}=8.7 \times$ $10^{20} \mathrm{~cm}^{-2}(5 \sigma)$ reached by Espada et al. (2011a). The black circle at the bottom left represents the beam size of $28^{\prime \prime} \times 28^{\prime \prime}$.

The brightest stellar structures within the pseudo-ring $\left(\mu_{\text {Cous } R}(\mathrm{CAHA})=25.5-26.5 \mathrm{mag} \operatorname{arcsec}^{-2}\right)$ are located within a distance of $r=1.5-2.0 \times r_{25}$ from the galaxy centre (i.e. approximately $3.5^{\prime}-4.7^{\prime}$ or $15.0-20.5 \mathrm{kpc}$ ). They are well defined and large to the north, thinner to the west and more diffuse to the south (see Fig. 2). The east region shows very diffuse emission and no clear sign of the pseudo-ring structure, making the latter a partially closed pseudo-ring. Despite the SB limit reached, the numerous stars in the field and their PSFs may play a relevant role by overlapping with any fainter emission at such low SB, mimicking non-existent extragalactic stellar traces (Trujillo \& Fliri 2016). In particular, this occurs in the eastern region where a few bright stars are located. However, the even deeper SB limit reached with the VST image has two immediate implications: one, the definition of certain regions of the pseudo-ring are greatly improved and two, the Galactic cirrus starts to become clearly visible at $28.5 \mathrm{mag} \operatorname{arcsec}^{-2}$, hindering the detection of features beyond the pseudo-ring at SBs fainter than this level (see Sect. 4.6).

\subsection{Disc and pseudo-ring relative orientation}

A visual inspection of the CAHA $2.2 \mathrm{~m}$ optical image suggested an apparent misalignment between the pseudo-ring and the galactic disc. In order to quantify it, we performed elliptical fittings to the pseudo-ring structure as well as to the isophotes of the galaxy from 20.2 to $26.4 \mathrm{mag} \mathrm{arcsec}^{-2}$ after removing the signatures of the close bright stars to avoid biased fittings.

The fittings of the innermost regions of the galaxy ( $\mu_{\text {Cous } R}=24.0 \mathrm{mag} \mathrm{arcsec}^{-2}$ or brighter $)$ were not reliable because of the strong influence of the spiral arms. Moreover, bright close stars contaminate the outer regions (fainter than $\mu_{\text {Cous } R}=24.0$ mag $\operatorname{arcsec}^{-2}$ ). Even after removing them, too few points are left making reliable fittings difficult.

However, the optical images clearly showed the centre of the galaxy (error below 1"). After fitting the pseudo-ring we found a shift of $12^{\prime \prime}(\sim 1.2 \mathrm{kpc}$, the approximate length of the bar)

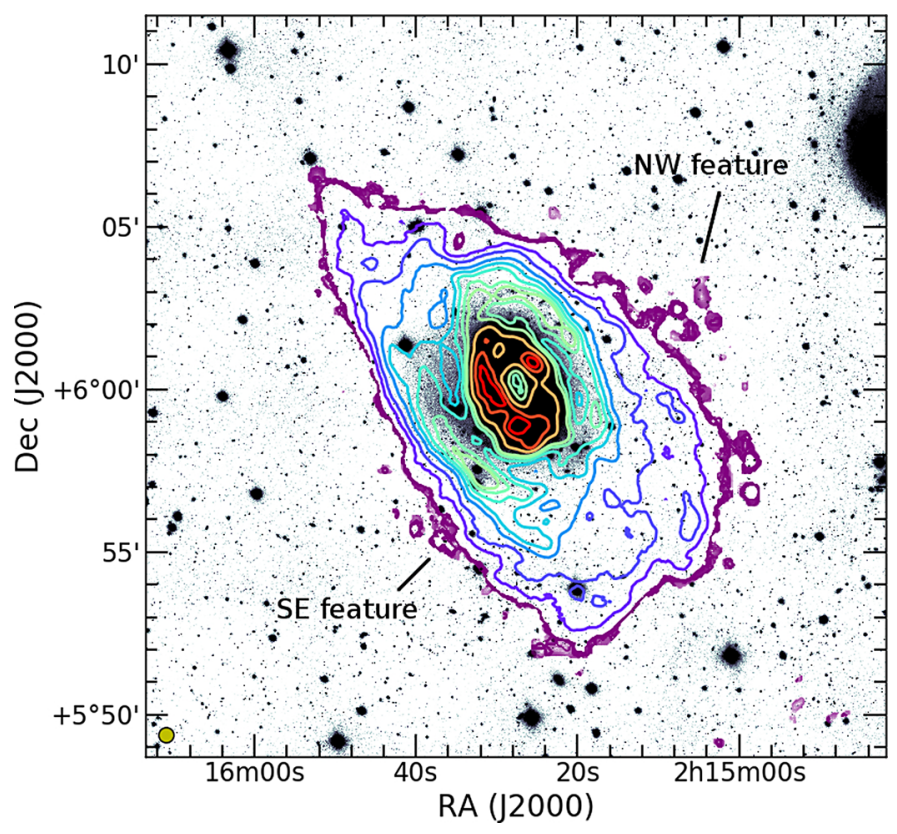

Fig. 6. Background: VST optical image of CIG 96 ranging from 26 to $28 \mathrm{mag} \mathrm{arcsec}^{-2}$. Foreground: HI cube integrated profile contours showing column densities of $0.6,7.1,14.1,28.2,42.3,56.5,70.6,80.4,105.8$, 127.0 and $141.1 \times 10^{20} \mathrm{~cm}^{-2}$. The yellow circle at the bottom left represents the beam size of $28^{\prime \prime} \times 28^{\prime \prime}$.

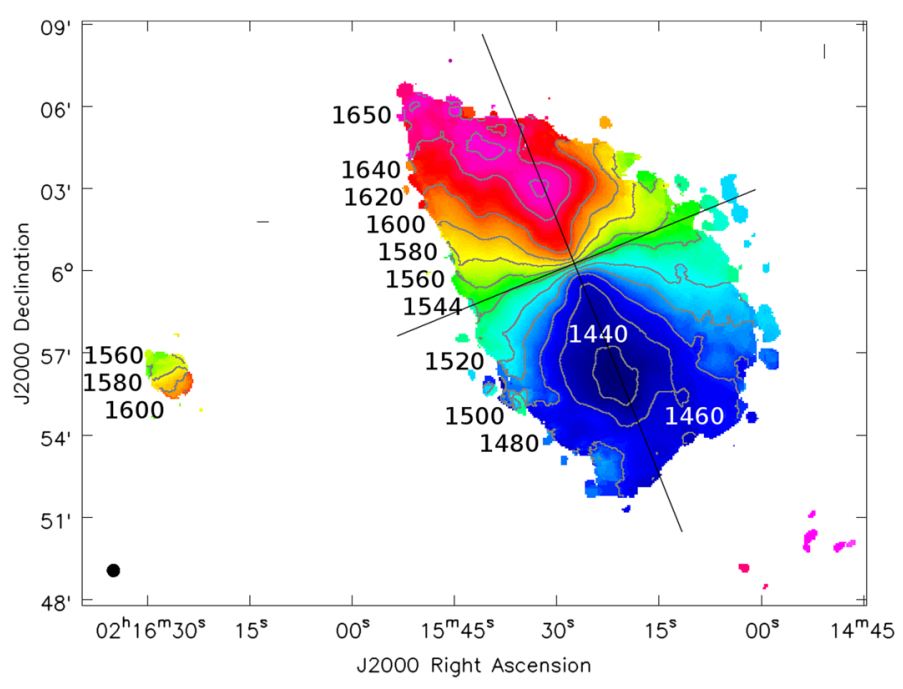

Fig. 7. HI velocity field map of CIG 96 and its companion after a $3.5 \sigma$ blanking (see Sect. 2.3). The black lines indicate the orientation of the major and minor axis $\left(\mathrm{PA}_{\text {maj }}=20^{\circ}\right.$ and $\mathrm{PA}_{\min }=110^{\circ}$, respectively) along which the position-velocity cuts have been performed (see Fig. 8). Grey contours represent the indicated velocities in $\mathrm{km} \mathrm{s}^{-1}$. The black circle at the bottom left represents the beam size of $28^{\prime \prime} \times 28^{\prime \prime}$.

between the centres of the pseudo-ring fitting and the disc and its orientation was $\mathrm{PA}_{\text {pseudo-ring fit }}=21.5^{\circ}$, similar to the $\mathrm{PA}$ of the major axis of the galaxy $\left(\mathrm{PA}_{\text {maj }}=20^{\circ}\right)$. We also de-projected the image assuming a disc inclination of $i_{\mathrm{CIG} 96}=46.59^{\circ}$ to confirm whether the pseudo-ring may be oval or in a different plane from the disc. We found the flattening or ellipticity of the pseudo-ring is of $0.04-0.05 \%$, that is, practically circular, suggesting it to be slightly oval if seen at almost the same inclination as the inner disc of the galaxy. 


\subsection{CIG 96 colour index image and optical features}

We analyse here the colour index image of CIG 96 and the distribution along the pseudo-ring (further discussed in Sect. 5.2) via CAHA1.23m $B$ and $R$ images converted to SDSS $g$ and $r$ magnitudes, respectively (see Sect. 2.4.2).

As a reference for the colour index values plotted in Fig. 9 left and central panels, we indicate the boundaries of the blue and red clouds from the SDSS $g-r$ optical colour-magnitude diagram. In particular we show the Green Valley interval of $(g-r)_{\mathrm{GV}}=0.60-0.75 \mathrm{mag}$ as defined by Walker et al. (2013) following the colour analysis by Strateva et al. (2001).

Qualitatively, we also note three striking features from the $g-r$ and optical images (see Fig. 9, left and central panels). The first feature is a diffuse arc in the east side of the pseudo-ring that almost closes it from north to south (Fig. 9, left panel, orange arrow); it is barely detectable (below $\sim 1.2 \sigma$ ) in any individual image further than a diffuse emission due to the heavy contamination of nearby stars. The second structure is also barely detectable (below $1.2 \sigma$ ) in any individual image despite there being no significant contamination by close stars in this region. It is located beyond the southern region of pseudo-ring, approximately $30 \mathrm{kpc}\left(\sim 5^{\prime}\right)$ from the galaxy centre (Fig. 9, left and centre panels, cyan arrow). The third structure is indicated with yellow crosses in the central panel of Fig. 9. This double structure has a SB of $\sim 26.0 \mathrm{mag} \operatorname{arcsec}^{-2}$ seems to connect the northern and southern inner parts of the galaxy with the western and eastern sides of the pseudo-ring, respectively.

Both in our VST image and in the DECaLS DR5 image, we detect a faint elongated (approximately $\sim 1^{\prime}$ long) and diffuse structure to the NE of CIG 96 (coordinates RA $=2^{\mathrm{h}} 15^{\mathrm{m}} 58.286^{\mathrm{s}}$, $\left.\operatorname{Dec}=+6^{\circ} 04^{\prime} 39.15^{\prime \prime}\right)$. It is located close to a bright star and barely a few kiloparsecs beyond the field of view covered by our $g-r$ image. As described further in Sect. 4.6, this structure lies on a region with a noticeable amount of background emission, mostly due to Galactic cirrus, and could therefore be part of it. However, we cannot rule out that this feature might be a tidal stream or a tidal disruption dwarf.

\subsubsection{Pseudo-ring colour index distribution}

We have studied the azimuthal variation of the colour index along the pseudo-ring by determining its median value in $33 \mathrm{cir}-$ cular non-overlapping apertures distributed in foreground starfree regions along its extent as shown in the central panel of Fig. 9, except for the NE region (PA in the range $38^{\circ}-70^{\circ}$ ) due to the lack of reliable optical emission in this arc. We defined the apertures over a de-projected image of CIG 96. For a better visualization, we have kept their spatial location and circular shape in the image presented in the previous figure, which is not de-projected. In order to discard any colour index changes in the pseudo-ring due to a gradient in the sky level, we determined the sky colour index of 62 regions set farther than the pseudoring, covering $360^{\circ}$ around CIG 96 and free of bright stars. These apertures show $g-r$ values between $\sim 0.2$ and $\sim 1.2$. In Fig. 10 we show the $g-r$ colour index distribution of all regions according to their PA and we find no colour index correlation between the apertures from the pseudo-ring and those from sky. However, as anticipated in the central panel of Fig. 9, we find a colour index change in between two PA ranges of the pseudo-ring. The 17 apertures of the $\mathrm{SE}$ arc (within $\mathrm{PA}=70^{\circ}-258^{\circ}$ ) show a median colour of $g-r=0.73 \mathrm{mag}$ (st.dev. $=0.15 \mathrm{mag}$ ), that is, a redder colour. Contrarily, the remaining 16 apertures of the NW arc (within $\mathrm{PA}=258^{\circ}-38^{\circ}$ ) show a median value of $g-r=0.31 \mathrm{mag}$

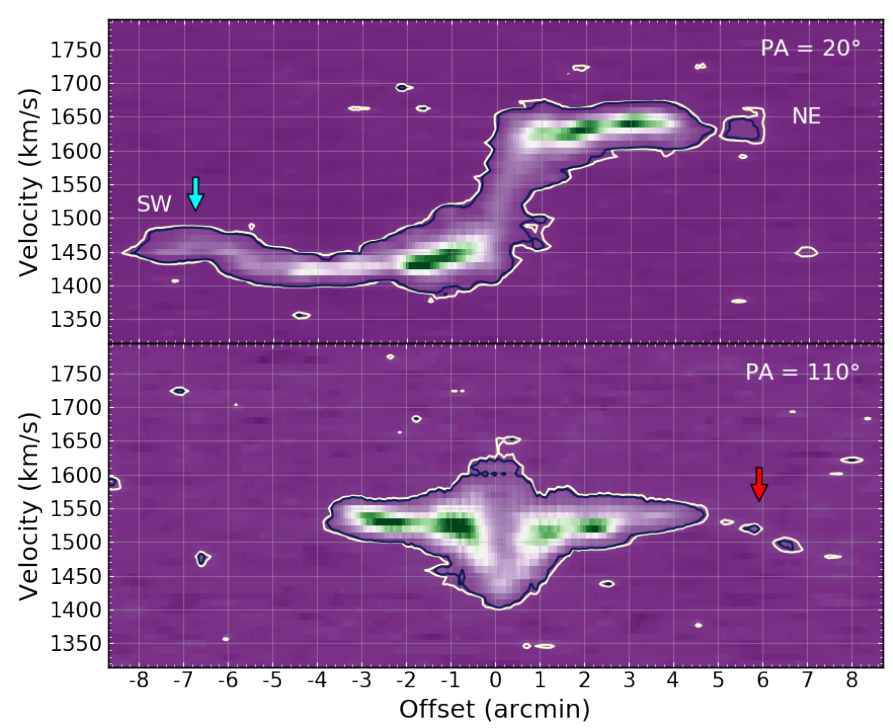

Fig. 8. Position-velocity cuts along the major axis (top panel, $\mathrm{PA}=20^{\circ}$ ) and minor axis (bottom panel, $\mathrm{PA}=110^{\circ}$ ) of $\mathrm{CIG} 96 \mathrm{HI}$ cube. The column density is $N_{\mathrm{HI}}(1 \sigma)=0.24 \times 10^{20} \mathrm{~cm}^{-2}$ and the white and black contours correspond to $3.5 \sigma$ and $5 \sigma$, respectively. The cyan arrow points to the SW region where the velocity increases by approximately $30-40 \mathrm{~km} \mathrm{~s}^{-1}$ (see Sect. 3.4). The red arrow points to the NW HI feature, the clumpy structure detected visible in channels $16-23$ of the HI cube (see Sect. 3.2). As a reminder, the beam resolution is of $28^{\prime \prime} \times 28^{\prime \prime}$.

(st.dev. $=0.11 \mathrm{mag}$ ), that is, a bluer colour, making the difference between the two regions of $g-r \simeq 0.4 \mathrm{mag}$.

\subsubsection{Radial cuts}

In order to compare the colour of the disc with the immediate pseudo-ring regions we computed radial profiles from individual $g$ and $r$ images. The right panel of Fig. 9 shows the de-projected $g$ image of CIG 96 together with the lines along which those were calculated.

These profiles are shown in Fig. 11, where the bulge (the first $2.5 \mathrm{kpc}$, Espada et al. 2011a), disc and pseudo-ring radii are marked as well. We selected the orientations due to the different structures crossed: disc, dust regions, arms, star-forming regions and thicker/thinner regions of the pseudo-ring. The profiles were then computed at PA of $6^{\circ}, 16^{\circ}, 30^{\circ}$ and $55^{\circ}$ and we will refer to them as PA6, PA16, PA30 and PA55, respectively.

To present the main results that these profiles yield, we have used a SB of $26.8 \mathrm{mag} \operatorname{arcsec}^{-2}$ in the SDSS $r$ band. At this depth, the disc size varies in a range of $R_{\text {disc }}=9.5-11 \mathrm{kpc}$, depending on the PA.

The gap between the disc and pseudo-ring is not constant either: in the regions where the pseudo-ring and the disc are well resolved, the gap has an approximate width of $\simeq 1 \mathrm{kpc}$. However, in regions where both the disc and pseudo-ring have a more diffuse emission, they prevent any reliable estimation of this separation.

The gap width, as well as its uncertainty, has a connection to the pseudo-ring dimensions: the more defined regions of the pseudo-ring have a width of $w_{\text {pseudo-ring }} \simeq 2 \mathrm{kpc}$ but it may rise up to $\sim 4 \mathrm{kpc}$ in some diffuse regions being hardly distinguishable from the disc.

Profile PA6 (green) shows red colours along the disc relative to the limit defined by the Green Valley strip. The peak at 

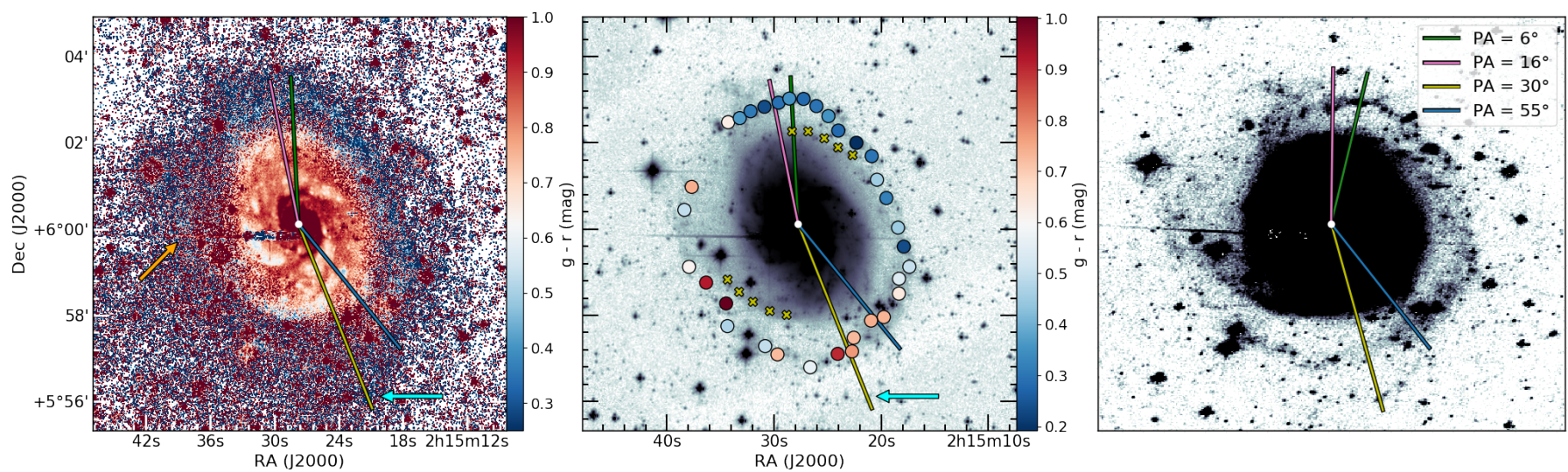

Fig. 9. Left panel: SDSS $g-r$ colour index image. Orange arrow points to the eastern arc of the pseudo-ring. Cyan arrow points to an optical feature to the south. Blue and red tones indicate colour index in magnitudes. The pink, blue and green lines indicate the directions (or PA) used to compute the four radial profiles discussed in Sect. 4.3.2; they are repeated in the other panels. Central panel: 33 circular apertures of $1.25 \mathrm{kpc}$ radius (12.7") located over star-free areas of the pseudo-ring, represented over the CAHA2.2m and CAHA1.23m combined optical image. A bluer/redder region indicates a bluer/redder $g-r$ colour index. The cyan arrow points again to the southern feature. Yellow marks indicate the connecting regions between the pseudo-ring and the inner parts of the galaxy. Right panel: de-projected SDSS $g$ CAHA1.23m image of CIG 96; the lines of the radial profiles have been reoriented to preserve the correct PA.

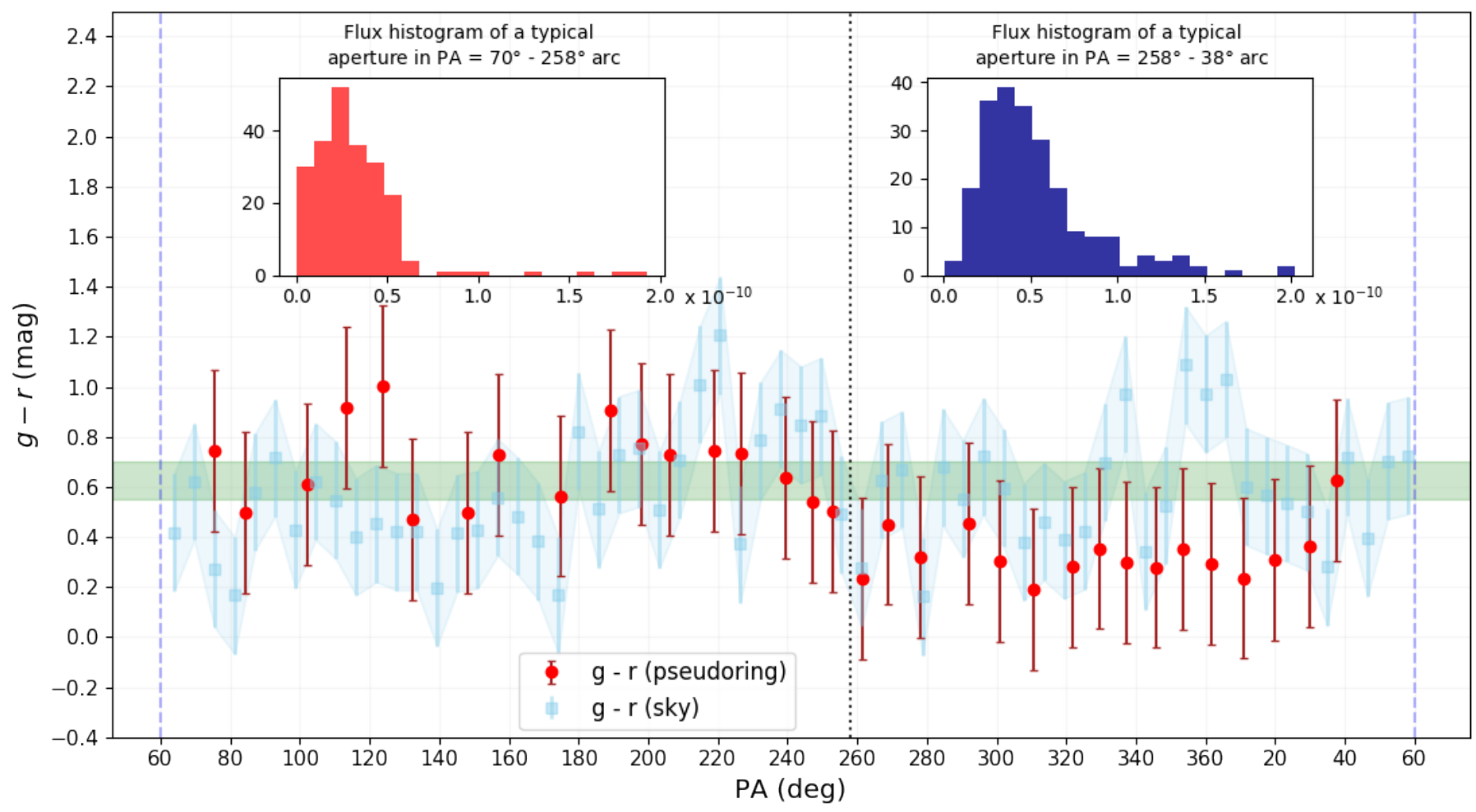

Fig. 10. SDSS $g-r$ colour index vs. PA along the pseudo-ring. The red dots represent the $g-r$ values obtained from the de-projected images. They are obtained by dividing $g$ median flux to the corresponding $r$ median flux of each aperture and converting these results to magnitudes. The pale blue dots correspond to the $g-r$ colour index measured at a distance of $r=29.5 \mathrm{kpc}\left(\sim 5^{\prime}\right)$ on the sky. The green stripe sets the Green Valley interval that separates the red cloud $(g-r>0.75 \mathrm{mag})$ from the blue cloud $(g-r<0.60 \mathrm{mag})$ as defined in 4.3 . The embedded figures correspond to typical flux $\times 10^{-10}$ histograms for two apertures from the SE region $\left(\mathrm{PA}=70^{\circ}-258^{\circ}\right.$, left panel $)$ and the $\mathrm{NW}$ region $\left(\mathrm{PA}=258^{\circ}-38^{\circ}\right.$, right panel) separated by the vertical doted grey line.

$\sim 7 \mathrm{kpc}$ corresponds to a foreground $\mathrm{star}\left(m_{r} \operatorname{SDSS}=19.65 \mathrm{mag}\right)$. The pseudo-ring shows blue colours in most of its extent along this PA, matching the star-forming region $(\sim 0.7 \mathrm{kpc}$ size $)$ present in this section of the cut, centred at a radius of approximately $12.5 \mathrm{kpc}$. The colour difference of the disc and the pseudo-ring at this PA is $\sim 0.4$ mag.

Profile PA16 (pink) also shows the difference in colours between the disc and the pseudo-ring. The disc shows a stable red colour throughout its whole extension $(g-r \simeq 0.7 \mathrm{mag}$ ). However, the pseudo-ring shows a colour gradient from $g-r \sim$ 0.7 to $0.1 \mathrm{mag}$ approximately, hence most of the pseudo-ring has blue colours. This profile was also aimed towards a large star forming region of $\sim 1.5 \mathrm{kpc}$ radius in the pseudo-ring and located at an approximate distance of $12 \mathrm{kpc}$, so such a blue colour is expected. However, there is no apparent cause for the colour change. 


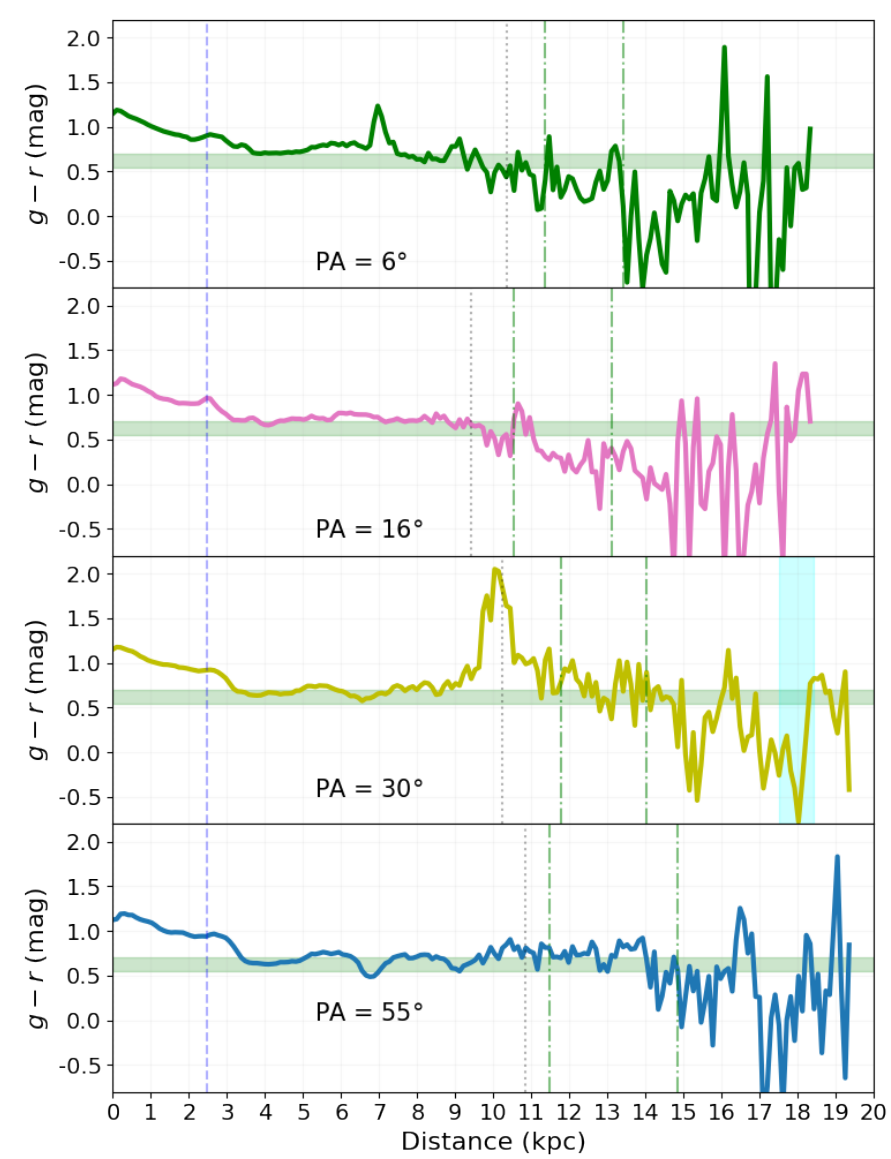

Fig. 11. Radial profiles obtained along four different orientations with PA of $6^{\circ}, 16^{\circ}, 30^{\circ}$ and $55^{\circ}$ (shown in Fig. 9, right panel) at the top, top-centre, bottom-centre and bottom panels, respectively. The horizontal green stripe represents the Green Valley in SDSS $g-r$ (see Sect. 4.3. The bulge, disc and pseudo-ring limits are measured at $26.8 \mathrm{mag} \mathrm{arcsec}^{-2}$. The bulge limit $(2.5 \mathrm{kpc})$ and the disc limit are shown as the light blue dashed and black dotted lines, respectively. The pseudoring variable inner and outer limits are defined in each panel by the green dot-dashed lines. The light cyan band in the $\mathrm{PA}=30^{\circ}$ panel sets the location of the optical feature to the $\mathrm{S}$ marked as a cyan arrow in the left panel of Fig. 9 and a magenta ellipse in Fig. 13.

Profile PA30 (yellow) shows a uniform disc colour within or right on the red edge of the Green Valley $(g-r \simeq 0.75 \mathrm{mag}$ ) consistent with the rest of the profiles. There is an exceptionally red peak at $10.5 \mathrm{kpc}$ that, unlike in the case of PA6 (produced by a star), is the result of a region with large quantities of dust. The orientation of the previous profiles missed these dusty inner regions of the galaxy, easily visible in the left panel of Fig. 9, left. PA30 crosses the pseudo-ring through an area of diffuse emission and the redder colour is consistent throughout its extension. The orientation of this profile was chosen to obtain also the colour of the southern feature of $\sim 1 \mathrm{kpc}$ in width located at $\sim 18 \mathrm{kpc}$ indicated with a red vertical stripe (also marked with a cyan arrow in Fig. 9, left panel). Despite the fact that the feature is surrounded by the sky, its location and the surrounding $0.5 \mathrm{kpc}$ show a clearly blue colour. We have not considered this feature as part of the pseudo-ring so its width estimation remains between 1.5 and $3.5 \mathrm{kpc}$ and its radius $\sim 14 \mathrm{kpc}$.

Profile PA55 (blue) shows a different behaviour along the disc. The mean colours are bluer than along the previous profiles; two regions that correspond to where the arms are crossed show very blue colours. This profile was selected to observe a

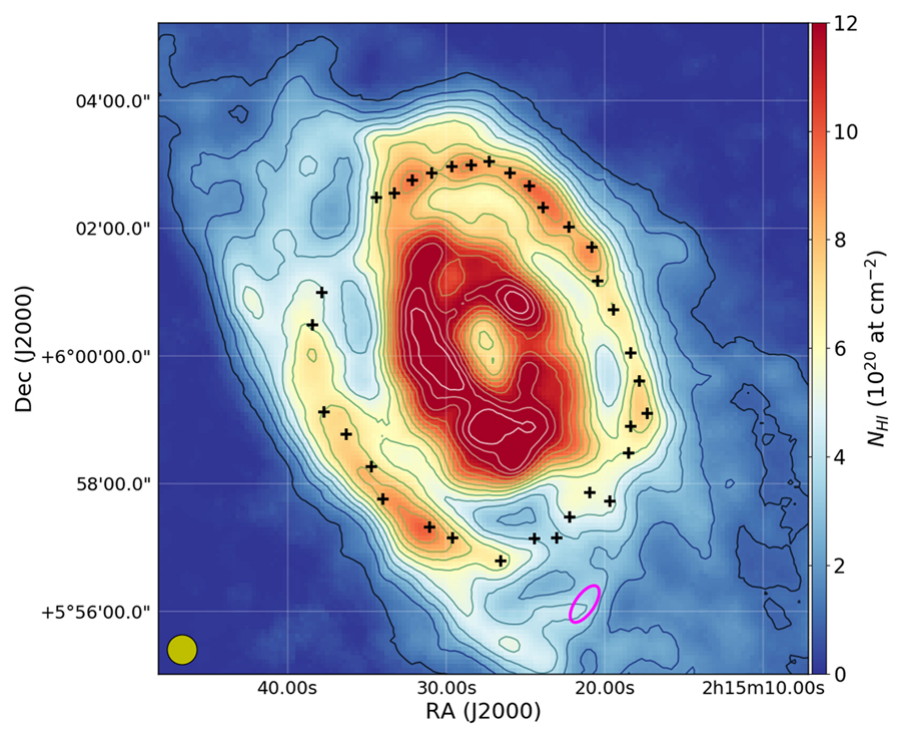

Fig. 12. Central $53 \times 53 \mathrm{kpc}(9 \times 9$ arcmin $)$ of the integrated HI emission map of CIG 96. Column density is indicated with a colour gradient. Contours indicate 1, 2, 3, 4, 5, 6, 7, 8, 9, 10, 11, 12, 13 and $14 \times 10^{20} \mathrm{at} \mathrm{cm}^{-2}$. The black crosses indicate the central position of the 33 apertures used to measure the colours of the pseudo-ring (see Sect. 4.3.1). The magenta ellipse indicates the position of the southern feature indicated with a cyan arrow in Fig. 9, left panel, the $g-r$ colour index image. The yellow circle at the bottom left indicates the HI image synthesized beam of $28^{\prime \prime} \times 28^{\prime \prime}$.

much more diffuse and broad region of the pseudo-ring (width up to $\sim 3.5 \mathrm{kpc}$ ). As in the disc, the pseudo-ring colour along this orientation is not homogeneous but it shows red colours ( $g-r \sim 0.8 \mathrm{mag}$ ) throughout most of its width. The farthest part of the pseudo-ring shows a steep change towards bluer colours, making it difficult to decipher whether it is an artifact of the sky or an existing structure with similar colour.

\subsection{Colour index and $\mathrm{HI}$ column density in the pseudo-ring}

The black crosses of Fig. 12 show the location of the apertures of the pseudo-ring on top of the HI 0th moment map. We find a remarkable spatial correlation between the optical pseudo-ring and the HI distribution, in agreement with Espada et al. (2011a). In Fig. 12 we indicate with a magenta ellipse the spatial location of the optical southern feature (shown in Fig. 9 left panel with a cyan arrow). It is too distant from the pseudo-ring $(\sim 4.1 \mathrm{kpc})$ as to confirm that both have a physical link and, unlike other starforming regions of the pseudo-ring, we find no increase of the $N_{\mathrm{HI}}$ in this region.

We have performed a detailed comparison between the pseudo-ring colour index $g-r$ and $N_{\mathrm{HI}}$ for each selected aperture. With this aim, we have scaled each one of them by subtracting the mean value of the 33 apertures and dividing them by their sigma value (Fig. 13, top panel). We observe an anticorrelation between $g-r$ and $N_{\mathrm{HI}}$ scaled values within $\mathrm{PA}=180^{\circ}-40^{\circ}$, i.e. bluer colours correspond to larger column densities. It is only broken in the range $\mathrm{PA}=90^{\circ}-180^{\circ}$, approximately, probably due to less reliable $g-r$ measurements in this side of the pseudo-ring, the most diffuse region. The anticorrelation is also confirmed in the bottom panel of Fig. 13 where we show $g-r$ as a function of $N_{\mathrm{HI}}$ : most of the bluer areas, mainly located in the NW side of the pseudo-ring $\left(\mathrm{PA}=260^{\circ}-40^{\circ}\right)$ show column densities of $8.5-13.5 \times 10^{20} \mathrm{~cm}^{-2}$, higher than most of the 

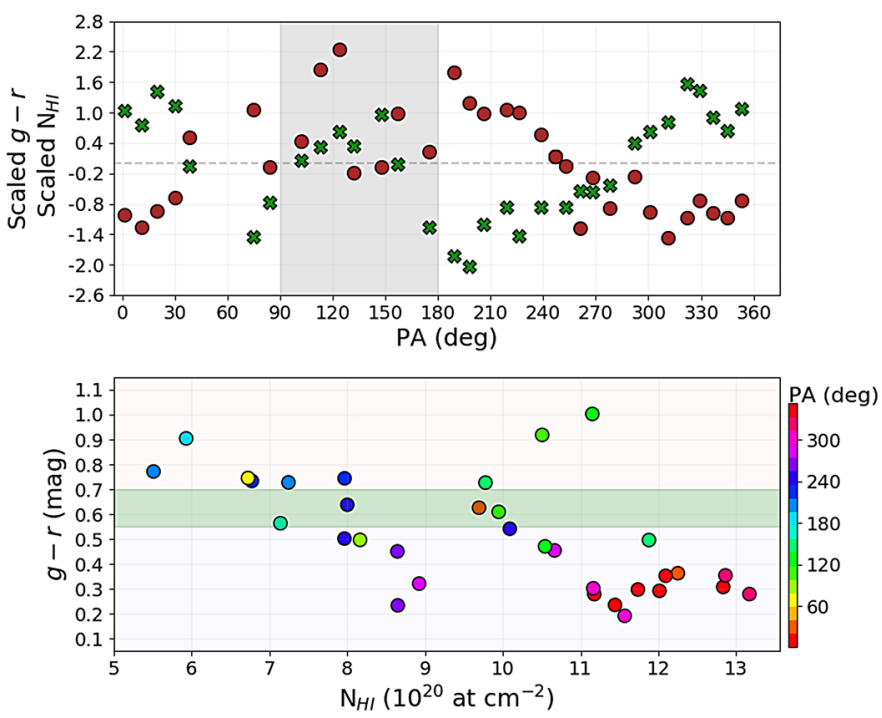

Fig. 13. Top panel: $g-r$ (brown circles) and $N_{\mathrm{HI}}$ (green crosses) scaled (mean subtracted, sigma divided) values in each of the 33 apertures. A grey dashed line has been drawn at $g-r=0$ for reference. Redder colours and higher $N_{\mathrm{HI}}$ are positive in this figure. Bottom panel: $g-r$ median colour index vs. $N_{\mathrm{HI}}$ measured in the 33 apertures traced over the pseudo-ring. The green horizontal stripe represents the Green Valley in SDSS $g-r$.

redder ones which show much lower levels instead. These results are discussed further in Sect. 5.2.

\subsection{Optical characteristics of the companion}

We aimed to observe any possible optical structures connecting CIG 96 and its companion (see Sect. 3.3). Given that the CAHA images have a field of view of $12^{\prime} \times 12^{\prime}$ (approximately $71 \mathrm{kpc} \times 71 \mathrm{kpc}$ ), it is only possible to studying any potential optical connection between the two galaxies with the VST $1^{\circ} \times 1^{\circ}$ image, as it provides continuous coverage across the $\sim 90 \mathrm{kpc}$ separation between them. Figure 14 shows a $10^{\prime} \times 10^{\prime}$ image centred between the two galaxies. At the current optical SB limit and in agreement with the HI map, we detect no sign of any stellar feature tracing any direct interaction between CIG 96 and its companion.

Focusing on its companion, our VST image shows that it consists of an elongated structure oriented with a PA of $35^{\circ}$ plus a spheroidal component. Although the HI resolution of our data prevents us from separating both optical components, the HI emission is slightly elongated along the same PA, within the resolution of our HI data $\left(28^{\prime \prime} \times 28^{\prime \prime}\right.$, see Fig. 5). Moreover, the HI kinematics shows the same orientation and is quite regular (within the limits given by the beam smearing), especially in the velocity range of $1560-1610 \mathrm{~km} \mathrm{~s}^{-1}$, where the main HI emission of the galaxy is found (see Fig. 7).

In order to determine whether the optical image of NGC 864 COM01 is compatible with a disc-bulge system, we proceeded in two steps. We inspected the model and residuals provided by DECam Legacy Survey (DECaLS DR5; Schlegel et al. 2015) and the model shows a good fit to the spheroidal component of the galaxy, with the residuals suggesting a blue and close to edge-on disc with a similar PA to the HI velocity field, as indicated above. Hence, from a morphological point of view it is compatible with a spiral galaxy with an $\mathrm{Sa}$ Sb type.

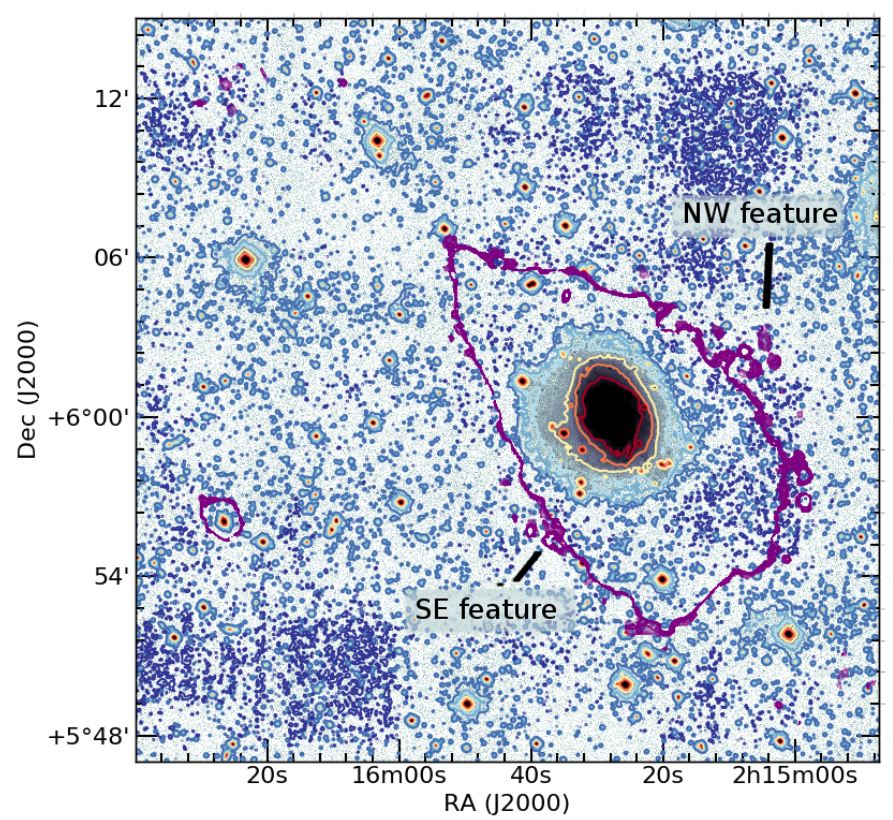

Fig. 14. VST image in SDSS $r$ band of a $28^{\prime} \times 28^{\prime}$ region covering both CIG 96 and its companion NGC 864 COM1 (indicated by the magenta contour to the east of the image). Optical contours are set in 24.0, 25.0, 26.0, 27.0, 27.5, 28.0 and $28.4 \mathrm{mag} \mathrm{arcsec}^{-2}$ (SDSS r), smoothed with a Gaussian kernel of 11 pixel radius. The purple contour marks the HI column density limit of the zeroth moment at $0.6 \times 10^{20} \mathrm{~cm}^{-2}$, as in Fig. 6.

As a next step we decided to perform a similar study to the analysis of the pseudo-ring of CIG 96, aiming to determine the colour of each of the two components and whether they are consistent with values found for spheroidal components and discs in spiral galaxies. In particular, we used SDSS $g$ and $r$ images from the DECaLS survey whose exquisite seeing allowed to produce a $g-r$ colour index image and measure the colours in the sky (median value is $0.49 \pm 0.07 \mathrm{mag}$ ) as well as in certain apertures, as shown in Fig. 15, central and bottom panels. The measures in these apertures show that the elongated structure is bluer $(0.33-0.45 \mathrm{mag})$ than the spheroidal one, which is redder (0.55-0.70 mag, right over the Green Valley range, see Fig. 10).

Lastly, we checked whether Sa-Sb galaxies exist with masses similar to that of the CIG 96 companion. We have used the value for the dynamical mass of NGC 864 COM01 as determined in Sect. $4.1\left(\sim 10^{9} M_{\odot}\right.$ which, as explained there, is a lower limit to the real one). According to Nair \& Abraham (2010) there exist $\mathrm{Sa}$ and $\mathrm{Sb}$ galaxies within such a mass range. Hence all these characteristics lead us to favour the possibility of NGC 864 COM01 being an Sa or Sb galaxy.

\subsection{Cirrus}

The possible large PSFs from close stars (whose radii reach several arcminutes, Trujillo \& Fliri 2016) and the necessity of a precise sky subtraction are key to discerning what may be artifacts from actual faint stellar components. However, there is another limiting factor at the current optical SB: the emission due to the presence of Galactic cold dust (Sandage 1976, confirmed with IRAS data by Neugebauer et al. 1984) known as cirrus in the Milky Way. This cirrus may cover large areas in the sky and, depending on the region, may start to be especially relevant from $\mu_{r \text { SDSS }} \simeq 25.5-26.0 \mathrm{mag} \operatorname{arcsec}^{-2}$ in the optical regime (Duc et al. 2015). 

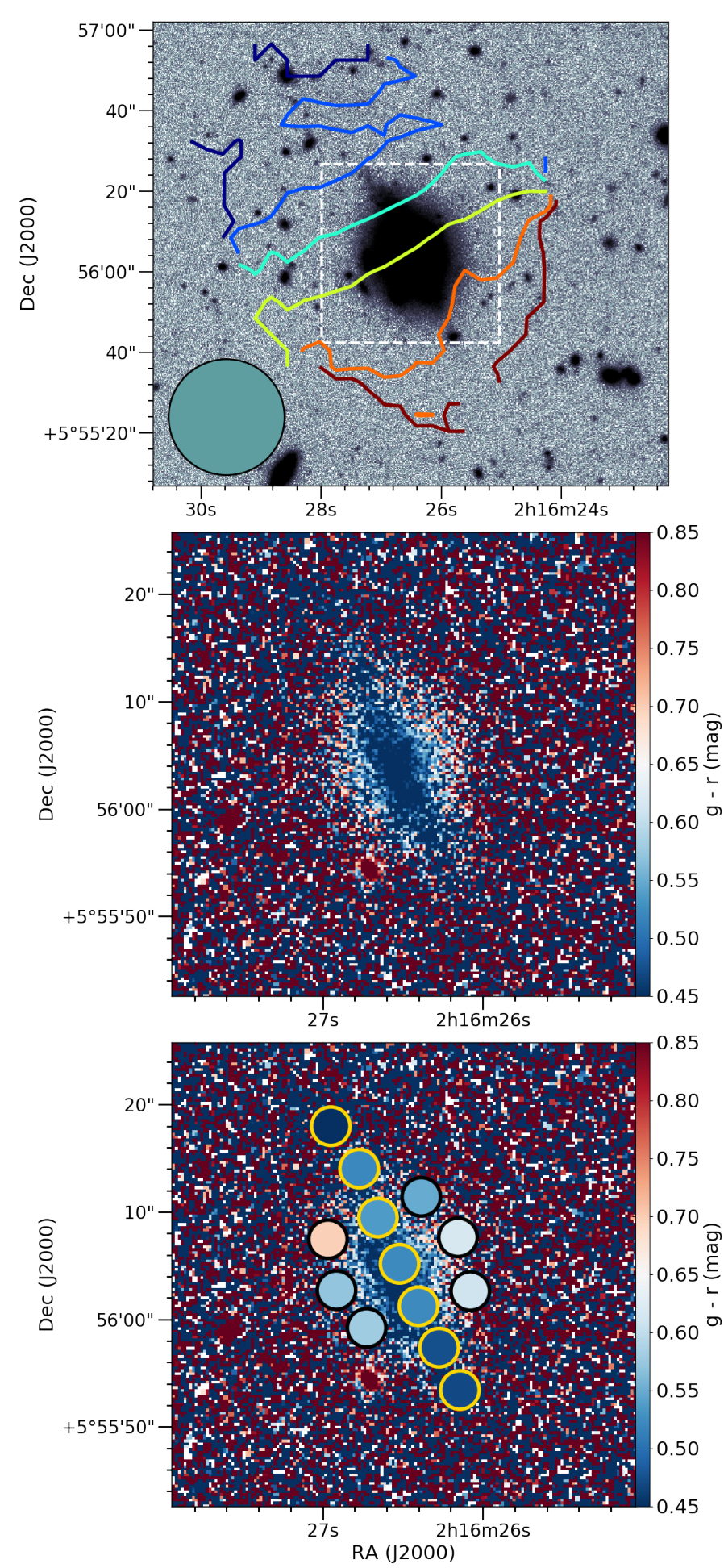

Fig. 15. Top panel: velocity contours of the blanked HI datacube from 1560 to $1610 \mathrm{~km} \mathrm{~s}^{-1}$ (in steps of $10 \mathrm{~km} \mathrm{~s}^{-1}$ ) on top of the VST image of the companion of CIG 96. The beam size of the HI data is indicated as a $28^{\prime \prime} \times 28^{\prime \prime}$ grey disc in the bottom left corner of the image. The white frame represents the central $45^{\prime \prime} \times 45^{\prime \prime}$ of the SDSS $g-r$ image of the bottom panel. Central panel: SDSS $g-r$ colour index image of the companion of CIG 96 built from the corresponding SDSS $g$ and $r$ images from DECaLS DR5 survey. Bottom panel: same image as in the central panel with the apertures used to measure the $g-r$ colour index. All apertures have a $1.75^{\prime \prime}$ radius. The yellow-edge apertures trace the colours of the elongated feature while the black-edge apertures show the colours of the galaxy.
As described in Sect. 2.5, we have used Planck857 and WISE3 images to identify the presence of cirrus in the field of CIG 96. Their optical resolutions are 4.2' and 6.5", respectively, that is, approximately 242 and 6 times lower than the $1.04 \mathrm{arcsec}_{\mathrm{pixel}}{ }^{-1}$ resolution of our CAHA images or 1260 and 30 times lower than our VST image, respectively.

The Planck857 image is a good indicator of cirrus; however, we cannot extract reliable conclusions in the field of CIG 96 due to its low spatial resolution. We used the central $40^{\prime} \times 40^{\prime}$ of the VST image to inspect the cirrus (see Fig. 16, top left panel). It shows that this area is populated with scattered emission visible from $\sim 28.5 \mathrm{mag} \operatorname{arcsec}^{-2}$. While this image is not enough to conclude whether that emission is associated with cirrus or not, we can confirm it does not show up in any other observation considered in this work.

In the Planck857 image (Fig. 16, bottom left panel), a $\sim 2 \sigma$ peak of emission (where $\sigma$ is the rms measured on the clean SE region) is visible in the central pixels where the galaxy is located. In the surrounding area and close to the noise level, there are extended areas to the east, northwest and southwest of CIG 96 which seem to match some of the emission observed with the VST image at $28.5 \mathrm{mag} \operatorname{arcsec}^{-2}$.

The lack of cirrus structures brighter than $28.5 \mathrm{mag} \mathrm{arcsec}^{-2}$ suggests they provide scarce (if any) contamination at brighter levels in our VST image, hence we set our detecion limit at $28.4 \mathrm{mag} \mathrm{arcsec}^{-2}$ in SDSS $r$ band. From the opposite point of view, the low resolution of Planck857 makes it pointless to use such an image to find any cirrus structure in our optical image (Fig. 16, bottom right panel). Should these exist, a positive matching between our VST and Planck857 images would require extremely large and bright structures, easily detectable in both images; however, we do not find such large structures, preventing the use of the Planck857 image in this case.

WISE3 emission is shown in the top right panel of Fig. 16 over the optical VST image. A quick glance at both Planck857 and WISE3 emission shows there is good correspondence between them, as expected. However, this is not the case for the optical-infrared images. Despite its higher resolution, the WISE3 image only matches some areas from the optical image, showing no significant emission nearby CIG 96.

Following the same procedure as Duc et al. (2015), we aimed to trace the Galactic cirrus in the neighbourhood of CIG 96 with our VST and the WISE3 images. After a careful revision of the reduction and calibration of the VST data, this image shows background emission from a SB level of $28.5 \mathrm{mag} \mathrm{arcsec}^{-2}$ and fainter, that partially coincides with the IR emission from the WISE3 image (see Fig. 16, top right panel). We cannot rule out an instrumental origin for some parts of the background emission of the field, yet the partial match between the VST and WISE3 images suggests that most of these structures are not undesired products of a deficient flat-field correction or scattered light but are actual Galactic cirrus, as expected in most latitudes from SB levels of $25 \mathrm{mag} \operatorname{arcsec}^{-2}$ or higher (Guhathakurta \& Tyson 1989; Cortese et al. 2010; Duc et al. 2015). Assuming that the emission belongs to the Galactic cirrus, reaching the very faint SB limit of $28.5 \mathrm{mag} \operatorname{arcsec}^{-2}$ or fainter is crucial to assert their detection, implying that shallower images might be missing them. However, we cannot rule out an instrumental origin for the emission with the current data, and further optical and IR images of the same field with the same or fainter SB levels are desirable to confirm the nature of the background structures. 

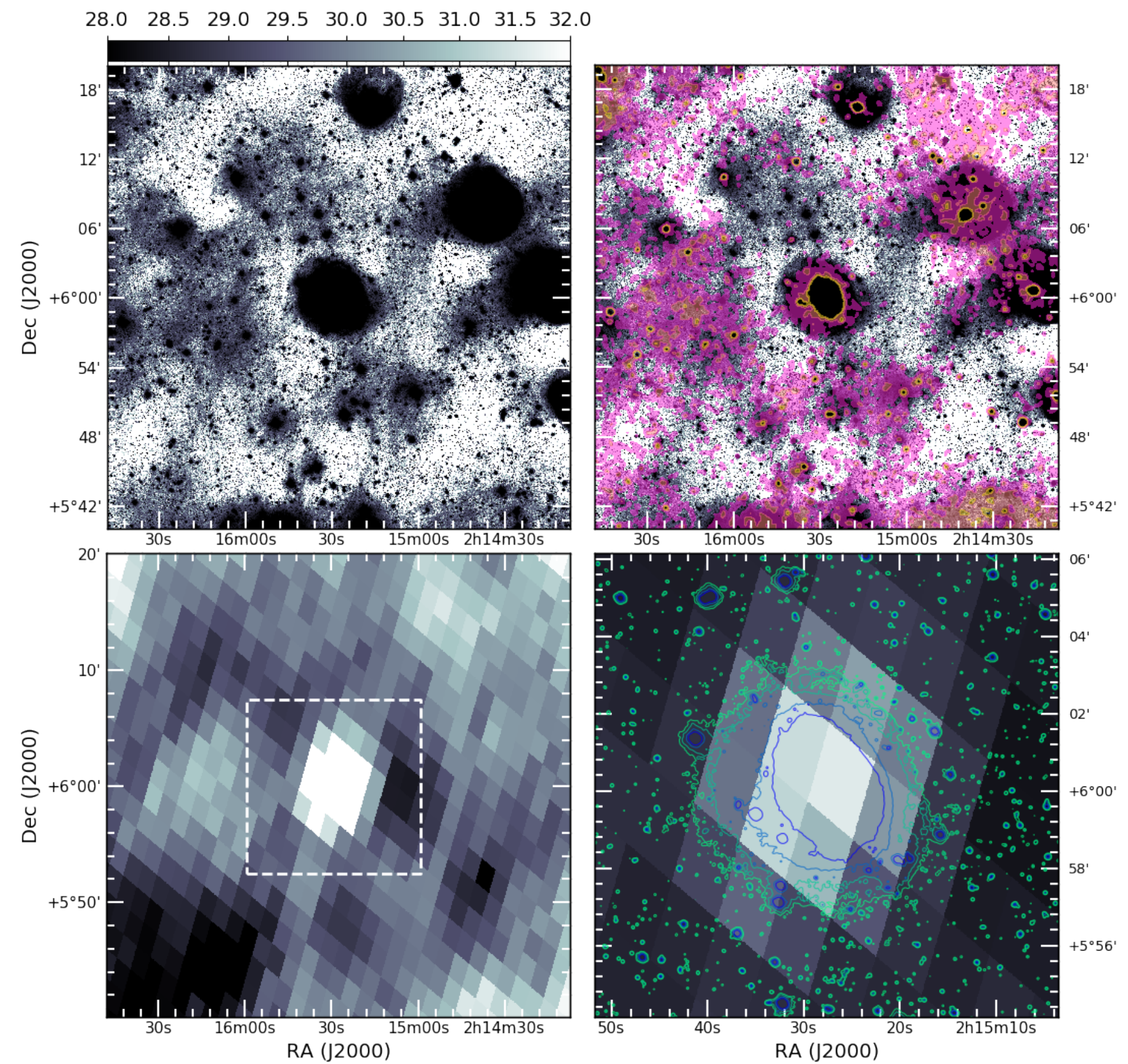

Fig. 16. Top left panel: central $40^{\prime} \times 40^{\prime}$ of the VST optical image in SDSS $r$ band of CIG 96. The SB is indicated on top of the figure in mag $\operatorname{~arcsec}^{-2}$ (SDSS $r$ band) favouring the levels that enhance the Galactic cirrus located in the field of our galaxy, which show up from $\mu_{r}$ SDSs $\simeq$ $28.5 \mathrm{mag} \operatorname{arcsec}^{-2}$. Top right panel: background, the same $40^{\prime} \times 40^{\prime}$ VST optical image as in top left panel; foreground, overlay of WISE band 3 $(12 \mu \mathrm{m})$ smoothed image contours (Gaussian kernel of 21 pixel radius). Bottom left panel: $40^{\prime} \times 40^{\prime}$ Planck857 GHz image of CIG 96 . The dashed white square indicates the approximate area selected for the bottom images. Bottom right panel: background, $12^{\prime} \times 12^{\prime} \mathrm{Planck} 857 \mathrm{GHz}$ image; foreground, 25.0, 26.0, 27.0, $27.5 \mathrm{mag} \operatorname{arcsec}^{-2}$ smoothed (Gaussian kernel of 7 pixel radius) optical contours from VST image (SDSS $r$ band).

In summary, Planck857 and WISE3 images show no signs of large, diffuse and faint structures over CIG 96 that might be interfering with our optical detection limits of the galaxy and its structures. However, we confirm a partial correspondence between the WISE3 image and the diffuse optical background emission in the surrounding field. The external (cirrus) or instrumental (flat-field correction) nature of the unmatched structures might be confirmed with further optical images of at least the same SB limit as our VST image. After a careful revision of the VST data processing, we must consider these background structures as a limiting factor to our images, setting the SB limit to $28.4 \mathrm{mag} \operatorname{arcsec}^{-2}$.

\section{Discussion of the optical and $\mathrm{HI}$ faint structures in the outskirts of CIG 96}

In this section we discuss the implications of the above results with respect to the close environment of CIG 96 as well as the origins of the different $\mathrm{HI}$ and optical features detected. 
Table 4. Companions of CIG 96 within $1 \mathrm{Mpc}$.

\begin{tabular}{ccccc}
\hline \hline Name & $\begin{array}{c}\text { Apparent } B \text { magnitude } \\
(\mathrm{mag})\end{array}$ & $\begin{array}{c}\text { Diameter (major axis) } \\
(\operatorname{arcmin} / \mathrm{kpc})\end{array}$ & $\begin{array}{c}\text { Projected distance } \\
(\operatorname{arcmin} / \mathrm{kpc} / \mathrm{direction})\end{array}$ & $\begin{array}{c}\text { Central velocity } \\
\left(\mathrm{km} \mathrm{s}^{-1}, \mathrm{LSRK}^{2}\right.\end{array}$ \\
\hline NGC 864 COM01 & 16.38 & $0.6 / 3.3$ & $15.24^{a} / 90^{a} / \mathrm{E}$ & $1578^{a}$ \\
HIPASS J0217+06 & 16.50 & $1.6 / 9.4$ & $40.03 / 235 / \mathrm{NE}$ & 1549 \\
UGC 01677 & 18.00 & $0.9 / 5.3$ & $76.70 / 450 / \mathrm{NW}$ & 1575 \\
UGC 01670 & 14.80 & $2.2 / 13.0$ & $83.73 / 495 / \mathrm{NW}$ & 1593 \\
UGC 01803 & 14.70 & $2.8 / 16.5$ & $89.26 / 527 / \mathrm{NE}$ & 1615 \\
\hline
\end{tabular}

Notes. Data from the NASA/IPAC Extragalactic Database (NED) unless stated otherwise. ${ }^{(a)}$ This work.

\subsection{The environment of CIG 96}

The two parameters that quantify the isolation degree of a galaxy from CIG are the local number density, $\eta_{k}$, that accounts for the number of neighbour galaxies within a radius of 0.5 or $1 \mathrm{Mpc}$ (depending on the criteria selected), and the tidal force estimation, $Q$, that quantifies how much the host is affected by its neighbourhood. According to the NASA/IPAC Extragalactic Database (NED), CIG 96 has five identified galaxies within a radius of $1 \mathrm{Mpc}$ : NGC $864 \mathrm{COM} 01$ (the aforementioned companion), HIPASS J0217+06, UGC 01677, UGC 01670 and UGC 01803. The properties of these neighbours are summarised in Table 4.

When taking the closest companion, NGC 864 COM01, the isolation time ${ }^{8}$ of CIG 96 results in $\sim 2.7$ Gyr. The rest of the targets, located farther away, yield isolation times of $\sim 11 \mathrm{Gyr}$ or higher than the age of the Universe, that is, they could not have interacted with CIG 96 in the past.

We used the methods from two previous works by Verley et al. (2007; hereafter V07) and Argudo-Fernández et al. (2013; hereafter A13) to calculate the isolation parameters of CIG 96 (see Sect. 1). V07 consider the first $k$ th similar sized neighbours within a $500 \mathrm{kpc}$ radius. In the case of CIG 96 only the closest two neighbours, i.e. NGC 864 COM01 and HIPASS J0217+06 are selected and the isolation parameters are $\eta_{k}^{\mathrm{V} 07}=0.877$ and $Q_{0.5}^{\mathrm{V} 07}=-1.981$. A13 extend the radius up to $1 \mathrm{Mpc}$, that is, they consider the five neighbours mentioned above, and calculate the isolation parameters with photometric and spectroscopic data. The $Q$ parameter of CIG 96 cannot be calculated with the photometric data because they contain no neighbour that violates the necessary criteria to measure the $Q$ between them and the host. However, the spectroscopic data allow to obtain the isolation parameters for CIG 96 being $\eta_{k}^{\mathrm{A} 13}=0.68$ and $Q^{\mathrm{A} 13}=-3.41$. This method is also used by Argudo-Fernández et al. (2014), where they focus on identifying the satellites around host galaxies from CIG, distinguish them from the Large Scale Structure (LSS) and quantify their effect over the host galaxy.

According to the spectroscopic data of A13, the position of CIG 96 is highlighted in Fig. 17 where lower values of $\eta_{k}$ and

\footnotetext{
8 As defined by Verdes-Montenegro et al. (2005) and in agreement with the isolation criteria, the isolation time estimates the minimum time a galaxy has been free of interactions with major companions. It is defined as follows: let $D$ be the diameter of CIG 96; the time that a hypothetical companion of $d=4 \times D$ size needs to cover a distance of $20 \times d$ by travelling at a typical "field" velocity of $150 \mathrm{~km} \mathrm{~s}^{-1}$ is $11.5 \mathrm{Gyr}$, that is, almost the age of the universe, implying no recent interaction. The closest companion NGC 864 COM01, travelling at a velocity of $33 \mathrm{~km} \mathrm{~s}^{-1}$ with respect to CIG 96, yields a minimum isolation time of $2.7 \mathrm{Gyr}$ while the other galaxies have never interacted with CIG 96.
}

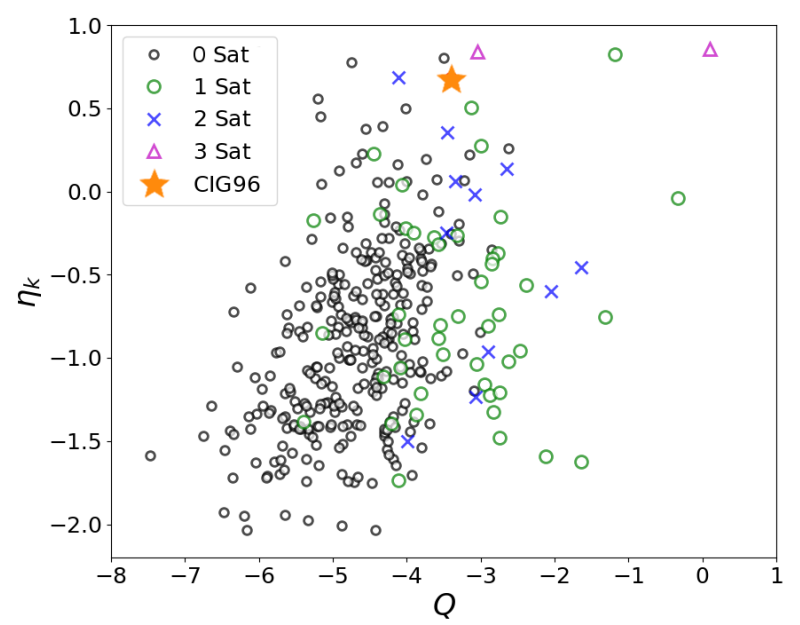

Fig. 17. Representation in the isolation parameters of the subsample generated with spectroscopic data of CIG galaxies as calculated by Argudo-Fernández et al. (2014; q.v. Fig. 8 of that work). Lower values of local number density $\eta_{k}$ and tidal force estimation $Q$ represent higher isolation. The position of CIG 96 is highlighted.

$Q$ represent higher isolation. The remaining galaxies shown for comparison correspond to the subsample of galaxies used in Argudo-Fernández et al. (2014) with $0,1,2$ or 3 dynamically linked satellites (physically bound neighbours, q.v. Fig. 5 in that work). The five closest companions of CIG 96 within $1 \mathrm{Mpc}$ are responsible for raising the $\eta_{k}$ parameter, whereas the $Q$ value is similar to galaxies with one or two satellites. However, CIG 96 has no satellite around it. This apparent contradiction reinforces a very relevant point: the isolation parameters are meant for statistical interpretations rather than to understand the environment of a particular galaxy. The physical interpretation of these parameters is that the populated environment of CIG 96 does not contain massive galaxies and, among all the neighbours, the closest companion included, none are affecting the evolution of CIG 96, as supported by all our observations. Despite the depth reached with our HI cube and optical images, we detect no signs of gaseous or stellar features between CIG 96 and its gas-rich companion (see Sects. 3.3 and 3.1) or any other more distant galaxies. The lack of detections supports the hypothesis of a long-lived isolation of CIG 96 where its asymmetrical HI distribution (see Fig. 5) may come from internal processes rather than an external agent with the exception of an absorbed small companion. HI asymmetries caused by major merger interactions are detectable within 0.4-0.7 Gyr (Holwerda et al. 2011), that is, approximately within one or two full rotations of CIG 96 ( $T_{\text {rot }}^{\mathrm{CIG} 96} \simeq 0.4 \mathrm{Gyr}$ ). However, the high isolation of the galaxy rules out encounters with major companions for at least the last $\sim 2.7$ Gyr. 
The Galactic cirrus is observed from a SB level of $\mu_{r \text { SDSS }}=28.5 \mathrm{mag} \mathrm{arcsec}^{-2}$ and fainter, affecting a remarkable area of the field around CIG 96. Infrared images may play a relevant role for the cirrus identification and future removal, therefore allowing for lower limits in SB to be reached. In this case, the two Planck857 and WISE3 images discussed do not manage to fully trace these structures at the sensitivity and spatial resolution required to remove them from the optical images. Since cirrus cannot be avoided by introducing any changes in the observational and data treatment strategy, they set an observational limit to our optical images, hampering the detection of fainter structures in polluted areas.

After reviewing the DECaLS DR5, a survey of similar characteristics with a SB limit of $\sim 28 \mathrm{mag} \mathrm{arcsec}^{-2}$ $\left(\sim 0.7 \mathrm{mag} \mathrm{arcsec}^{-2}\right.$ brighter than our image from VST), we did not spot clear signs of the structures we detect in our VST image. While we cannot fully rule out an instrumental origin, most of the faint structures surrounding CIG 96 only show up from $\sim 28.5 \mathrm{mag} \mathrm{arcsec}^{-2}$ and fainter, suggesting that very faint SB levels are necessary to detect them, possibly being missed by slightly shallower images. We do confirm though the detection of the small stellar feature indicated in the left and central panels of Fig. 9 (cyan arrow), a candidate ultra-diffuse galaxy (UDG).

\subsection{Origins of the features of CIG 96}

HI features. For decades it has been known that the HI in spiral galaxies frequently shows asymmetries and lopsidedness (Baldwin et al. 1980; Richter \& Sancisi 1994). However, the origin of such features in the isolated galaxies from CIG is unknown. The asymmetries of CIG 96 represent an excellent study case and have motivated all the observations and discussion of this work.

As described in Sects. 3.2 and 3.4 and shown in Figs. 4, 5 and 8, we observe two external HI clouds, the NW and SE HI features, which do not seem to belong to the main HI body of CIG 96 and a region with a remarkable receding velocity in the outermost SW region. The NW HI feature is a clumpy, almost continuous cloud spread throughout $\sim 70 \mathrm{~km} \mathrm{~s}^{-1}$ and connected to the galaxy approximately along the direction of its minor axis. This connection may be traced down to channels 24 and 25 (1560 and $1570 \mathrm{~km} \mathrm{~s}^{-1}$, respectively), where the HI cloud and the galaxy join showing no perturbation in the velocity field. The SE HI feature consists of a few gaseous clouds located in the same spatial location and spread along $\sim 40 \mathrm{~km} \mathrm{~s}^{-1}$. However, the lack of any noticeable perturbation over the outermost HI of CIG 96 suggest this feature has had little (if any) effect on the eastern side of the galaxy. It is also important to note that neither of these two features show any optical counterpart down to our detection limits.

The most distant HI region located at about $\sim 7^{\prime}(\sim 41 \mathrm{kpc})$ from the centre along the direction of the major axis (indicated with a cyan arrow in Fig. 8) shows a receding velocity that is $\sim 30-40 \mathrm{~km} \mathrm{~s}^{-1}$ above the expected velocity $\left(\sim 1430 \mathrm{~km} \mathrm{~s}^{-1}\right)$ of this region. Espada et al. (2005) proposed this region as a possible kinematically detached clump from the galaxy. The zeroth moment of our HI cube (Fig. 5) and the P/V cuts over the major axis show such a kinematical detachment as well as small and scattered unresolved HI clouds. However, they do not provide evidence of a physical detachment in the SW region (indicated with the cyan arrow in Fig. 8, top panel), which is larger than any of the small HI clouds nearby. The inspection of the firstmoment map suggests the farthest-south region might be warped but this would imply an external agent like a minor merger or the accretion of gas (Bournaud et al. 2005; Jog \& Combes 2009) that we do not find at our column density limit of $N_{\mathrm{HI}}=8.9 \times 10^{18} \mathrm{~cm}^{-2}$. For this reason, we cannot rule out the cold gas accretion below the already low column density reached as a candidate to explain such features.

The P/V profile along the minor axis of CIG 96 (Fig. 8) shows emission in a wide gradient of velocities, going up to $+85 \mathrm{~km} \mathrm{~s}^{-1}$ in the receding side and down to $-145 \mathrm{~km} \mathrm{~s}^{-1}$ in the approaching side with respect to its central velocity. Beam effects may contribute to such dispersion (Bosma 1978) by introducing part of the surrounding emission at different velocities. However, with the current resolution of $28^{\prime \prime} \times 28^{\prime \prime}$, this effect can only explain dispersions up to approximately $\pm 60-70 \mathrm{~km} \mathrm{~s}^{-1}$. Counter-rotating gas clouds may also contribute to the high velocity dispersion but we do not detect any signatures of such features in the major axis, where they would likely be visible. An outflow or infall of extraplanar gas of different speeds may also explain the wide range of velocities but we do not detect any signs of either of these in any channel of the map.

Accretion of cold HI clouds. High- and intermediate-velocity HI clouds around the Milky Way may reach masses of $10^{7} M_{\odot}$. However, the clouds of the Local Group have smaller typical total masses of $M_{\mathrm{HI}}^{\text {cloud }} \simeq 10^{5}-10^{6} M_{\odot}$ (Wakker et al. 1999) and they are expected to be several orders of magnitude below the total mass of their host galaxies.

With respect to CIG 96, from our HI cube we compute a total HI mass of $M_{\text {CIG } 96} \approx 9.77 \times 10^{9} M_{\odot}$. The two NW and SE HI features have total masses of $\sim 3.1$ and $1.6 \times 10^{6} M_{\odot}$, respectively, close to the detection limit achieved with our HI cube $\left(0.7 \times 10^{6} M_{\odot}\right)$. The NW HI feature meets the HI mass, spatial distribution, and velocity range criteria to consider it a possible infalling cloud that overlaps with CIG 96 in channels 23-25 (velocities $1550-1570 \mathrm{~km} \mathrm{~s}^{-1}$ ).

As discussed by Scott et al. (2014), these clouds are not expected to fall on an extension of the rotation curve. This reinforces the idea that the previously discussed SW side of the galaxy is likely to be the warped edge of the HI disc instead of an accreted HI cloud.

Pseudo-ring colour, column densities and minor mergers. $\mathrm{HI}$ is disrupted more easily than the faint optical halo substructures, which may live longer (Peñarrubia et al. 2005) than the $\sim 1.5-3$ Gyr established by the quantified isolation criteria (see Sect. 5.1). The external pseudo-ring of CIG 96 is HI rich except for its southern and NE sides where the gas is scarce. The pseudo-ring colours in the southern side are clearly redder than in the rest of the ring, in particular in the bluer northern and NW sides where SF is taking place in a number of scattered regions according to their blue colours. This colour difference agrees with GALEX NUV and FUV results discussed by Espada et al. (2011a).

The star-forming regions with high UV emission match the bluer regions of the N-NW side of the pseudo-ring, and consistently show column densities above $8.5 \times 10^{20} \mathrm{~cm}^{-2}$. These are higher than the redder regions which coincide with areas where the HI seems disturbed and show much lower HI column densities, as shown in the bottom panel of Fig. 13. The $N_{\mathrm{HI}}$ is measured with a beam larger than the size of the optical regions. Once resolved, we would expect to achieve an even higher $N_{\mathrm{HI}}$ level in the bluest regions, reaching the nominal SF value of $10^{21} \mathrm{~cm}^{-2}$. Also, the anticorrelation observed between the scaled $g-r$ and $N_{\mathrm{HI}}$ seems to break in the $70^{\circ}-180^{\circ}$ arc. This is the eastern side of the pseudo-ring where the emission is very diffuse so any correlation or anticorrelation between $g-r$ and $N_{\mathrm{HI}}$ in this area is uncertain. 
Among the mechanisms that involve the SF enhancement or quenching as well as HI asymmetries are the minor mergers with low-mass dwarf galaxies. On the one hand, one or a few recent minor mergers with HI-rich small galaxies (wet mergers) are expected to leave clear HI footprints tracing such events, let alone orbiting stellar structures (Martínez-Delgado et al. 2009) or SF enhancement in the areas where the merging occurred: i.e., bluer colours would be expected in the southern and NE areas instead of the redder colours we find. Also, these mergers could have occurred in the more diffuse and incomplete regions of the pseudo-ring but, again, footprints of such events are missing. On the other hand, any minor mergers with one or a few HI-poor companions (dry mergers) might explain the stripped gas as well as the consequent SF quenching (even extinction) of the NE and southern regions. However, we find no optical or gaseous signature in any of these sections and the zeroth and first moments indicate no link between the possible warp in the southern arc of the pseudo-ring. One or various possible encounters with ultra-diffuse galaxies (UDG) of large mass-tolight ratios (van Dokkum et al. 2016) might be responsible for the SF in the pseudo-ring. The only candidate to UDG lies to the south of the galaxy (see Figs. 9 and 11) but we detect no signs of interaction between this feature and the galaxy at the SB level reached with our observations.

The tidal footprints from older minor mergers $(t>0.7 \mathrm{Gyr}$, Holwerda et al. 2011) might have disappeared within one or two galaxy revolutions. Should they have left any optical counterparts that might still be visible, they would be expected to be weak and diffuse, such as those detected in the outskirts of other nearby galaxies (with no isolation classification) by Morales et al. (2018) at similar SB. Except for possible UDG mentioned before, we do not detect any other candidates or tidal streams brighter than our SB limit. However, the limiting SB level of $\mu_{r}$ SDSS $=28.5 \mathrm{mag} \operatorname{arcsec}^{-2}$ prevents the detection of further potential candidates fainter than this limit, that is, based solely on these optical observations, we may not discard the hypothesis that one or more minor mergers may be responsible for the asymmetries of the galaxy and the colour index variation in the pseudo-ring, nor the possibility that faint optical counterparts to the HI features might exist. Consequently, old minor mergers remain as possible candidates to explain the stellar and gaseous features of CIG 96.

Age of the pseudo-ring of CIG 96. The blue colour of $g-r$ representations is a good tracer of the age of the stellar population in the optical regime. Schawinski et al. (2009) propose three different models to discuss age according to the $g-r$ colour observed: model 1 assumes an instantaneous burst of SF with an exponential decay of $100 \mathrm{Myr}$; model 2 considers an instantaneous burst of SF with instantaneous decay of $10 \mathrm{Myr}$; lastly, model 3 assumes a constant SFR.

The colour nature of the pseudo-ring of CIG 96 does not fit with the age estimations of models 1 and 2 because so recent SF would either require nearby companions powerful enough to trigger it, or a transfer of gas from the inner parts towards the pseudo-ring in a lower period of time than the dynamical timescale of the galaxy. We do not detect signs of any of these requirements, leading us to consider model 3 as a more likely scenario. According to the latter, the continuous and slow ingestion of gas from the central parts of the galaxy into the pseudo-ring might explain a constant SF during at least 1 Gyr.

Additionally, Espada et al. (2011a) found that the outer parts of CIG 96 have ultraviolet colours of FUV-NUV= 0.1-0.2 $\mathrm{mag}_{\mathrm{AB}} \operatorname{arcsec}^{-2}$. According to this interval FUV-NUV, the low-metallicity model proposed by Smith et al. (2010) fits with the expected lower metallicity in the outskirts of a galaxy. However, it yields ages lower than $100 \mathrm{Myr}$ for the pseudo-ring. We discard the option of a companion since we would expect to detect it further than just its influence on the outskirts.

Origin of the pseudo-ring of CIG 96. The rings located 2-2.5 times the radius of the bar of the galaxy are labelled as outer rings (ORs; Buta 2017). Outer rings are typical in barred galaxies and their origin is gas accumulation in the outer Lindblad resonance (OLR; Schommer \& Sullivan 1976).

On the one hand, we find no signatures in either the optical or HI observations that suggest a collisional origin, in agreement with its high isolation level. On the other hand, the OLR is located at a distance slightly beyond twice the bar radius (Athanassoula et al. 1982). In the case of CIG 96, the bar and pseudo-ring optical radius are $\sim 2 \mathrm{kpc}\left(\sim 22^{\prime \prime}\right)$ and $\sim 14 \mathrm{kpc}$ $\left(\sim 145^{\prime \prime}\right)$, respectively, that is, the pseudo-ring is located over four times farther than the bar, making such a distance too large to be considered an OLR of the bar, as discussed by Espada et al. (2005).

An oval shape of the bright inner disc of the spiral galaxy might be a more reliable source to explain the origin of the pseudo-ring, as discussed briefly by Espada et al. (2011a). These non-axisymmetric kinematical features can produce disturbances on the motions of gas clouds located in the outer HI disc, resulting in complete or partial resonance rings (Schwarz 1981; Verdes-Montenegro et al. 1995; Buta \& Combes 1996). In agreement with what is expected to be found in these resonance rings, the pseudo-ring of CIG 96 has a symmetric shape and it is partially defined, showing diffuse optical emission and low HI column densities in regions to the NE and SW.

An apparent misalignment between the galaxy centre and the pseudo-ring might be suggested by external isophotes of CIG 96 (q.v. Figs. 2 and 14). This is confirmed by the different elliptical fittings of the pseudo-ring that consistently show its centre lies $\sim 1.2 \mathrm{kpc}\left(\sim 12^{\prime \prime}\right)$ to the south of the galaxy centre. However, the diffuse optical eastern side of the pseudo-ring and the high contamination of bright stars around the galaxy prevent a reliable global isophotal fitting analysis and further discussion on this topic.

The nearby stars also prevent us from clearly resolving the two elongated and faint arms or extensions that depart from the north and south of the disc. However, both the UV data analysed by Espada et al. (2011a) and our CAHA optical images (q.v., Figs. 2 and 9, central panel, yellow crosses) show that the northern and southern extensions connect the disc and the outermost regions of the galaxy where, respectively, they join the western and eastern sides of the pseudo-ring.

Detection of fainter signatures of interactions. Up to date, the studies performing deep optical observations of nearby galaxies mostly detect the brightest stellar features located in their outskirts. Standard $\Lambda$-CDM cosmological simulations of galactic halos (e.g. Bullock \& Johnston 2005; Johnston et al. 2008; Cooper et al. 2010) show that a large portion of the debris from old and minor mssergers may be fainter than $\sim 30 \mathrm{mag} \mathrm{arcsec}^{-2}$. For this reason, we can confirm that we do not detect signatures of these minor interactions in the outskirts of CIG 96 down to the SB and $N_{\mathrm{HI}}$ limits reached with our optical and HI observations, respectively, yet there may be unveiled features lying at fainter SB levels. Currently, for galaxies located close to the Milky Way, the only approach for detecting these very faint remnants of interactions consists of performing stellar density maps of evolved stellar components (e.g. RGB stars) in the halos of these galaxies (e.g. the PISCeS 
survey and study of Cen A and NGC 253; Crnojević et al. 2016 2018). These studies reach SB limits of 32-34 mag arcsec ${ }^{-2}$, yet this technique is not feasible for more distant galaxies like CIG 96 and farther with the current ground-based telescopes.

\section{Conclusions}

The AMIGA project uses a sample that shows the most symmetric HI integrated profiles when compared to any other sample, even field galaxies. However, some of its members present very asymmetric profiles as well as other features whose origins remain unknown. If large asymmetries are mostly generated by interactions, the lopsidedness of an isolated galaxy such as CIG 96 should not be observed. CIG96 is an isolated galaxy of the AMIGA sample that shows a $16 \%$ asymmetry in its HI profile as well as an actively star forming external pseudo-ring detected in the optical, UV and HI regimes. Our deep optical and HI observations have yielded unprecedented detail of the stellar and gas components of the galaxy and its outskirts. The wide field of view of $1^{\circ} \times 1^{\circ}$ of VST telescope and the wavelet-filtered $21-\mathrm{cm}$ data from VLA/EVLA telescope allowed us to reach a maximum SB and column density level of $\mu_{r \mathrm{SDSS}}=28.7 \mathrm{mag} \operatorname{arcsec}^{-2}$ and $N_{\mathrm{HI}}=8.9 \times 10^{18} \mathrm{~cm}^{-2}(5 \sigma$, beam size of $28^{\prime \prime} \times 28^{\prime \prime}$ ), respectively. The optical data reveal the detailed structure of the pseudo-ring as well as a gradient in its colour index. Moreover, the HI data show previously undetected features very close to the galaxy. Next, we present the main conclusions of this work:

Down to these limits, we do not find any gaseous or stellar connection between CIG 96 and any galaxy in its close environment, including its closest, largest, and HI-rich companion NGC 864 COM01, located 15.2' ( 90 kpc) to the east (projected distance) that may be a close to edge-on $\mathrm{Sa}$ or Sb galaxy, as the optical and HI properties of the system suggest. Scattered Galactic cirrus shows up from $28.5 \mathrm{mag} \operatorname{arcsec}^{-2}$ (SDSS $r$ band) in the surroundings of the galaxy and prevents any positive detection of further faint optical features beyond this depth.

We find two low-mass HI features $\left(\sim 10^{6} M_{\odot}\right)$ located to the NW and SE edges of the galaxy (the NW and SE HI features). The NW HI feature is visible along a number of immediate channels of the HI cube and depicts a low column density cloud $\left(N_{\mathrm{HI}}^{\mathrm{NW}} \simeq 6.5 \times 10^{19} \mathrm{~cm}^{-2}\right)$ connected with CIG 96 slightly to the $\mathrm{N}$ of its minor axis. We think that the SE HI feature, however, is a series of thin, small and spatially aligned clouds $\left(N_{\mathrm{HI}}^{\mathrm{SE}} \simeq 4.9 \times 10^{19} \mathrm{~cm}^{-2}\right)$ that stand out in the zeroth moment. The individual channels of the HI cube show that the different clouds that compose this feature are not connected to one another and show no direct effect on the immediate gas of the disc edge despite its close proximity.

We find a colour index difference of $g-r \simeq 0.4$ mag between two sides of the partially complete pseudo-ring $\left(\mathrm{PA}_{\text {redder }}=\right.$ $70^{\circ}-258^{\circ}$ and $\mathrm{PA}_{\text {bluer }}=258^{\circ}-38^{\circ}$ ) that cannot be assigned to any instrumental effect. No environmental cause (external gas accretion or minor merger) has been identified in our data as to explain such a change in the colour index. The outermost star-forming regions detected with NUV and FUV images from GALEX coherently match the blue regions of the pseudo-ring, which also show $N_{\mathrm{HI}}$ values close to $10^{21} \mathrm{~cm}^{-2}$, the nominal SF value. The cause for the higher concentration of $N_{\mathrm{HI}}$ in certain areas of the pseudo-ring is still to be found.

We have reviewed different SF models based on the FUV-NUV and $g-r$ colours to determine the approximate age of the pseudo-ring of CIG 96. We may discard a short lived pseudo-ring ( $\sim 100 \mathrm{Myr}$ or younger) caused by a very recent encounter with either a similar-sized galaxy (the isolation criteria discard them) or a small galaxy (we would expect to see the merger remains); instead, they favour an older pseudo-ring $(\sim 1 \mathrm{Gyr})$.

Despite the fact that bars are usually relevant candidates to play a critical role in the secular evolution of the outskirts of a galaxy by leading the matter to concentrate in the OLR, such is not the case for CIG 96. First, we do not find any significant matter concentration in the OLR of CIG 96 and, second, the pseudo-ring is located at almost double the radius of the expected location of the OLR based on the bar size. For these reasons, we cannot consider a bar-driven accumulation of matter in the OLR as the pseudo-ring origin. Either an oval distortion or old, elongated arms expelled from the inner parts of the disc are more fitting explanations of the origin of the faint, distant (from the galaxy centre) and circular pseudo-ring of CIG 96. Star-forming regions are expected in secular evolution and they may be triggered by external factors such as encounters with smaller and fainter infalling HI clouds. However, we do not find evidence of any external event that may explain the star-forming regions of the pseudo-ring of CIG 96. Their origin remains unknown.

The lack of any remarkable tidal features or other stellar components leads us to consider that, on the one hand, any major encounter with similar sized galaxies must have never occurred, as guaranteed by the isolation times and criteria (see Sect. 5.1); on the other hand, any possible minor merger must have taken place before the last two revolutions of CIG96 $(t>0.8 \mathrm{Gyr}$, approximately), allowing the footprints of such encounters to disappear within such a time.

Acknowledgements. P.R.M. and all the coauthors thank the referee for the careful reading and valuable suggestions provided in the report, which have helped to improve this paper significantly. P.R.M. is funded by the project AMIGA4GAS project and the FPI Grant AYA2011-30491-C02-01 by the Ministry of Economy and Competitiveness of Spain. P.R.M., L.V.M., J.B.H., M.J., M.F.L., S.S.E., J.G.S. acknowledge support from the grant AYA2015-65973C3-1-R (MINECO/FEDER, UE). P.R.M acknowdledges Víctor Terrón for his assistance during the calibration of the optical data. P.R.M. and the rest of the coauthors acknowledge Tom Jarrett for providing the WISE image used in this work; I.Trujillo for the discussion with respect to the deep optical observations and techniques; Monika Petr-Gotzens (ESO) for the help, follow-up and good advices provided with the VST observations and Enrique Pérez for his help with the dynamical mass estimations. L.V.M. acknowledges discussions with Alberto Fernández-Soto about the CAHA1.23m data. J.I.P. acknowledges financial support from the Spanish Ministerio de Economía y Competitividad under grant AYA2013-47742-C4-1-P, and from Junta de Andalucía Excellence Project PEX2011-FQM-7058. This publication makes use of data products from the Wide-field Infrared Survey Explorer, which is a joint project of the University of California, Los Angeles, and the Jet Propulsion Laboratory/California Institute of Technology, funded by the National Aeronautics and Space Administration. The National Radio Astronomy Observatory (NRAO) Karl G. Jansky Very Large Array (VLA) is a facility of the National Science Foundation operated under cooperative agreement by Associated Universities, Inc.. This research made use of Astropy, a community-developed core Python package for Astronomy (Astropy Collaboration 2013, 2018) and of APLpy, an open-source plotting package for Python (Robitaille \& Bressert 2012). We used the NASA/IPAC Extragalactic Database (NED), operated by the Jet Propulsion Laboratory, California Institute of Technology, under contract with the National Aeronautics and Space Administration. IRAF is distributed by the National Optical Astronomy Observatories, which are operated by the Association of Universities for Research in Astronomy, Inc., under cooperative agreement with the National Science Foundation.

\section{References}

Ahn, C. P., Alexandroff, R., Allende Prieto, C., et al. 2012, ApJS, 203, 21 Argudo-Fernández, M., Verley, S., Bergond, G., et al. 2013, A\&A, 560, A9 Argudo-Fernández, M., Verley, S., Bergond, G., et al. 2014, A\&A, 564, A94 Astropy Collaboration (Robitaille, T. P., et al.) 2013, A\&A, 558, A33 
Astropy Collaboration (Price-Whelan, A. M., et al.) 2018, AJ, 156, 123 Athanassoula, E., Bosma, A., Creze, M., \& Schwarz, M. P. 1982, A\&A, 107, 101 Baldwin, J. E., Lynden-Bell, D., \& Sancisi, R. 1980, MNRAS, 193, 313 Bijaoui, A.1991, The Wavelet Transform, Data Analysis Workshop, 3

Bosma, A. 1978, PhD Thesis, Groningen Univ., the Netherlands

Bosma, A. 2017, in Outskirtsof Galaxies, eds. J. H. Knapen, J. C. Lee, \& A. Gil de Paz, Astrophys. Space Sci. Lib., 434, 209

Bosma, A., \& Freeman, K. C. 1993, AJ, 106, 1394

Bournaud, F., Combes, F., Jog, C. J., \& Puerari, I. 2005, A\&A, 438, 507

Bullock, J. S., \& Johnston, K. V. 2005, ApJ, 635, 931

Buta, R., \& Combes, F. 1996, Fund. Cosmic Phys., 17, 95

Buta, R., Vasylyev, S., Salo, H., \& Laurikainen, E. 2005, AJ, 130, 506

Buta, R. J., Sheth, K., Athanassoula, E., et al. 2015, ApJS, 217, 32

Buta, R. J., Vasylyev, S., Salo, H., \& Laurikainen, E. 2017, MNRAS, 471, 4027

Cooper, A. P., Cole, S., Frenk, C. S., et al. 2010, MNRAS, 406, 744

Cortese, L., Bendo, G. J., Isaak, K. G., Davies, J. I., \& Kent, B. R. 2010, MNRAS, 403, L26

Courteau, S., Cappellari, M., de Jong, R. S., et al. 2014, Rev. Mod. Phys., 86, 47

Crnojević, D., Sand, D. J., Spekkens, K., et al. 2016, ApJ, 823, 19

Crnojević, D., Sand, D., Spekkens, K., et al. 2018, Meeting Abstr., 231, 36

de Vaucouleurs, G., de Vaucouleurs, A., \& Corwin, Jr., H. G. 1991, Third Reference Catalogue of Bright Galaxies (New York, USA: Springer)

Duc, P.-A., Cuillandre, J.-C., Karabal, E., et al. 2015, MNRAS, 446, 120

Erroz-Ferrer, S., Knapen, J. H., Font, J., et al. 2012, MNRAS, 427, 2938

Espada, D., Bosma, A., Verdes-Montenegro, L., et al. 2005, A\&A, 442, 455

Espada, D., Muñoz-Mateos, J. C., Gil de Paz, A., et al. 2011a, ApJ, 736, 20

Espada, D., Verdes-Montenegro, L., Huchtmeier, W. K., et al. 2011b, A\&A, 532, A117

Fernández Lorenzo, M., Sulentic, J., Verdes-Montenegro, L., et al. 2012, A\&A, 540, A47

Fernández Lorenzo, M., Sulentic, J., Verdes-Montenegro, L., et al. 2014, ApJ, 788, L39

Fitzpatrick, E. L. 1999, PASP, 111, 63

Fouque, P., Durand, N., Bottinelli, L., Gouguenheim, L., \& Paturel, G. 1990, A\&AS, 86, 473

Grossmann, A., \& Morlet, J. 1985, in Mathematics+Physics, Lecture on Recent Results, ed. L. Streit (Singapore: World Scientific)

Guhathakurta, P., \& Tyson, J. A. 1989, ApJ, 346, 773

Haynes, M. P., van Zee, L., Hogg, D. E., Roberts, M. S., \& Maddalena, R. J. 1998, AJ, 115, 62

Högbom, J. A. 1974, A\&AS, 15, 417

Holwerda, B. W., Pirzkal, N., Cox, T. J., et al. 2011, MNRAS, 416, 2426

Iodice, E., Spavone, M., Capaccioli, M., et al. 2017, ApJ, 839, 21

Jog, C. J., \& Combes, F. 2009, Phys. Rep., 471, 75

Johnston, K. V., Bullock, J. S., Sharma, S., et al. 2008, ApJ, 689, 936

Jones, M. G., Espada, D., Verdes-Montenegro, L., et al. 2018, A\&A, 609, A17

Karachentseva, V. E. 1973, Astrofizicheskie Issledovaniia Izvestiya Spetsial'noj Astrofizicheskoj Observatorii, 8

Kazantzidis, S., Bullock, J. S., Zentner, A. R., Kravtsov, A. V., \& Moustakas, L. A. 2008, ApJ, 688, 254

Kazantzidis, S., Zentner, A. R., Kravtsov, A. V., Bullock, J. S., \& Debattista, V. P. 2009, ApJ, 700, 1896

Kerr, F. J., \& Lynden-Bell, D. 1986, MNRAS, 221, 1023

Kormendy, J., \& Kennicutt, Jr., R. C. 2004, ARA\&A, 42, 603

Kornreich, D. A., Haynes, M. P., Jore, K. P., \& Lovelace, R. V. E. 2001, AJ, 121 1358

Leon, S., \& Verdes-Montenegro, L. 2003, A\&A, 411, 391

Leon, S., Meylan, G., \& Combes, F. 2000, A\&A, 359, 907
Leon, S., Cortes, P. C., Guerard, M., et al. 2016, A\&A, 586, A70

Lisenfeld, U., Verdes-Montenegro, L., Sulentic, J., et al. 2007, A\&A, 462, 507

Lupton, R. 2005, http://classic.sdss.org/dr5/algorithms/ sdssUBVRITransform. html

Martínez-Delgado, D., Peñarrubia, J., Gabany, R. J., et al. 2008, ApJ, 689, 184

Martínez-Delgado, D., Pohlen, M., Gabany, R. J., et al. 2009, ApJ, 692, 955

Martínez-Delgado, D., D’Onghia, E., Chonis, T. S., et al. 2015, AJ, 150, 116

McFarland, J. P., Verdoes-Kleijn, G., Sikkema, G., et al. 2013, Exp. Astron., 35, 45

McGlynn, T., \& Scollick, K. 1994, in Astronomical Data Analysis Software and Systems III, eds. D. R. Crabtree, R. J. Hanisch, \& J. Barnes, ASP Conf. Ser., 61,34

McMullin, J. P., Waters, B., Schiebel, D., Young, W., \& Golap, K. 2007, in Astronomical Data Analysis Software and Systems XVI, eds. R. A. Shaw, F. Hill, \& D. J. Bell, ASP Conf. Ser., 376, 127

Miville-Deschênes, M.-A., Duc, P.-A., Marleau, F., et al. 2016, A\&A, 593, A4

Morales, G., Martínez-Delgado, D., Grebel, E. K., et al. 2018, A\&A, 614, A143

Muldrew, S. I., Croton, D. J., Skibba, R. A., et al. 2012, MNRAS, 419, 2670

Nair, P. B., \& Abraham, R. G. 2010, ApJS, 186, 427

Neugebauer, G., Habing, H. J., van Duinen, R., et al. 1984, ApJ, 278, L1

Peñarrubia, J., Martínez-Delgado, D., Rix, H. W., et al. 2005, ApJ, 626, 128

Planck Collaboration I. 2014, A\&A, 571, A1

Portas, A., Scott, T. C., Brinks, E., et al. 2011, ApJ, 739, L27

Richter, O. G., \& Sancisi, R. 1994, A\&A, 290

Roberts, M. S. 1962, AJ, 67, 437

Roberts, M. S. 1975, in Radio Observations of Neutral Hydrogen in Galaxies, eds. A. Sandage, M. Sandage, \& J. Kristian (The University of Chicago Press), 309

Robitaille, T., \& Bressert, E. 2012, Astrophysics Source Code Library [record ascl:1208.017]

Sabater, J., Verdes-Montenegro, L., Leon, S., Best, P., \& Sulentic, J. 2012, A\&A, 545, A 15

Sandage, A. 1976, AJ, 81, 954

Savage, B. D., \& Mathis, J. S. 1979, ARA\&A, 17, 73

Schawinski, K., Virani, S., Simmons, B., et al. 2009, ApJ, 692, L19

Schlegel, D. J., Blum, R. D., Castander, F. J., et al. 2015, Meeting Abstr., 225, 07

Schommer, R. A., \& Sullivan, III., W. T. 1976, Astrophys. Lett., 17, 191

Schwarz, M. P. 1981, ApJ, 247, 77

Scott, T. C., Sengupta, C., Verdes Montenegro, L., et al. 2014, A\&A, 567, A56

Sengupta, C., Scott, T. C., Verdes Montenegro, L., et al. 2012, A\&A, 546, A95

Smercina, A., Bell, E. F., Price, P. A., et al. 2018, ApJ, 863, 152

Smith, B. J., Giroux, M. L., Struck, C., \& Hancock, M. 2010, AJ, 139, 1212

Stoughton, C., Lupton, R. H., Bernardi, M., et al. 2002, AJ, 123, 485

Strateva, I., Ivezić, Ž., Knapp, G. R., et al. 2001, AJ, 122, 1861

Toomre, A. 1977, ARA\&A, 15, 437

Trujillo, I., \& Fliri, J. 2016, ApJ, 823, 123

Trujillo, I., Roman, J., Filho, M., \& Sánchez Almeida, J. 2017, ApJ, 836, 191 van Dokkum, P. G., Abraham, R., Merritt, A., et al. 2015, ApJ, 798, L45 van Dokkum, P., Abraham, R., Brodie, J., et al. 2016, ApJ, 828, L6

Venhola, A., Peletier, R., Laurikainen, E., et al. 2017, A\&A, 608, A142

Verdes-Montenegro, L., Bosma, A., \& Athanassoula, E. 1995, A\&A, 300, 65

Verdes-Montenegro, L., Sulentic, J., Lisenfeld, U., et al. 2005, A\&A, 436, 443

Verdes-Montenegro, L., Del Olmo, A., \& Sulentic, J. 2010, ASP Conf. Ser., 421

Verley, S., Leon, S., Verdes-Montenegro, L., et al. 2007, A\&A, 472, 121

Wakker, B. P., Howk, J. C., Savage, B. D., et al. 1999, Nature, 402, 388

Walker, L. M., Butterfield, N., Johnson, K., et al. 2013, ApJ, 775, 129 